\title{
Hardy Spaces and bmo on Manifolds with Bounded Geometry
}

\author{
Michael TAYLOR ${ }^{1}$
}

Abstract. We develop the theory of the "local" Hardy space $\mathfrak{h}^{1}(M)$ and JohnNirenberg space $\operatorname{bmo}(M)$ when $M$ is a Riemannian manifold with bounded geometry, building on the classic work of Fefferman-Stein and subsequent material, particularly of Goldberg and Ionescu. Results include $\mathfrak{h}^{1}-$ bmo duality, $L^{p}$ estimates on an appropriate variant of the sharp maximal function, $\mathfrak{h}^{1}$ and bmo-Sobolev spaces, and action of a natural class of pseudodifferential operators, including a natural class of functions of the Laplace operator, in a setting that unifies these results

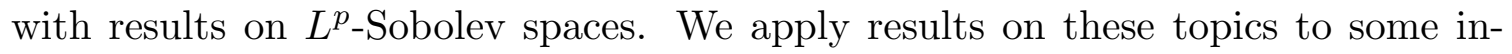
terpolation theorems, motivated in part by the search for dispersive estimates for wave equations.

\section{Introduction}

The theory of the Hardy space $H^{1}\left(\mathbb{R}^{n}\right)$ of functions on Euclidean space $\mathbb{R}^{n}$ and its connection to the John-Nirenberg space $\mathrm{BMO}\left(\mathbb{R}^{n}\right)$ were highly developed in the classic paper [FS]. One characterization of $H^{1}\left(\mathbb{R}^{n}\right)$ given there goes as follows.

$$
H^{1}\left(\mathbb{R}^{n}\right)=\left\{f \in L_{\text {loc }}^{1}\left(\mathbb{R}^{n}\right): \mathcal{G} f \in L^{1}\left(\mathbb{R}^{n}\right)\right\},
$$

where

$$
\mathcal{G} f(x)=\sup _{0<r<\infty} \sup _{\varphi \in \mathcal{F}}\left|\int \varphi_{r}(x-y) f(y) d y\right|,
$$

with

$$
\varphi_{r}(x)=r^{-n} \varphi\left(r^{-1} x\right),
$$

and $\mathcal{F}$ a collection of functions that can be rather flexible. For example, one could take

$$
\mathcal{F}=\left\{\varphi \in C_{0}^{1}\left(B_{1}\right):\|\nabla \varphi\|_{L^{\infty}} \leq 1\right\}
$$

or one could take

$$
\mathcal{F}=\{\varphi\},
$$

\footnotetext{
${ }^{1}$ Math Subject Classifications. 58J40, 58J05, 46E35

Key Words and Phrases. Hardy space, bmo, pseudodifferential operators, Riemannian manifolds, bounded geomerty

Acknoledgments. Work supported by NSF grant DMS-0456861
} 
consisting of a single function $\varphi \in \mathcal{S}\left(\mathbb{R}^{n}\right)$ such that $\int \varphi(x) d x=1$. (Cf. Theorem 11 of [FS], and also [Sem].) Such flexibility in specifying $\mathcal{F}$ is itself a useful tool in the study of $H^{1}\left(\mathbb{R}^{n}\right)$.

One of the major results of [FS] was the proof of the duality

$$
H^{1}\left(\mathbb{R}^{n}\right)^{\prime}=\operatorname{BMO}\left(\mathbb{R}^{n}\right)
$$

where the right side is the John-Nirenberg space, defined by

$$
\operatorname{BMO}\left(\mathbb{R}^{n}\right)=\left\{f \in L_{\text {loc }}^{1}\left(\mathbb{R}^{n}\right): f^{\#} \in L^{\infty}\left(\mathbb{R}^{n}\right)\right\}
$$

(modulo constants), where

$$
f^{\#}(x)=\sup _{B \in \mathcal{B}(x)} \frac{1}{V(B)} \int_{B}\left|f(y)-f_{B}\right| d y,
$$

with

$$
\mathcal{B}(x)=\left\{B_{r}(x): 0<r<\infty\right\}
$$

$B_{r}(x)$ being the ball centered at $x$ of radius $r$, and

$$
f_{B}=\frac{1}{V(B)} \int_{B} f(y) d y
$$

There are variants giving the same space. For example, one could use cubes containing $x$ instead of balls centered at $x$ (as did [JN] and [FS] in their original works), and one could replace $f_{B}$ in (1.7) by $c_{B}$, chosen to minimize the integral. The flexibility afforded by the equivalence of these different characterizations is again useful (as we will see, in a related context, in $\S 3$ ).

A number of variants of these spaces have been studied. In $[\mathrm{G}]$ "local" spaces $\mathfrak{h}^{1}\left(\mathbb{R}^{n}\right)$ and $\operatorname{bmo}\left(\mathbb{R}^{n}\right)$ were defined, as follows.

$$
\mathfrak{h}^{1}\left(\mathbb{R}^{n}\right)=\left\{f \in L_{\text {loc }}^{1}\left(\mathbb{R}^{n}\right): \mathcal{G}^{b} f \in L^{1}\left(\mathbb{R}^{n}\right)\right\},
$$

where

$$
\mathcal{G}^{b} f(x)=\sup _{0<r \leq 1} \sup _{\varphi \in \mathcal{F}}\left|\int \varphi_{r}(x-y) f(y) d y\right|
$$

with $\varphi_{r}$ and $\mathcal{F}$ as described above. It was shown in [G] that (1.5) implies

$$
\mathfrak{h}^{1}\left(\mathbb{R}^{n}\right)^{\prime}=\operatorname{bmo}\left(\mathbb{R}^{n}\right)
$$


where $\operatorname{bmo}\left(\mathbb{R}^{n}\right)$ is defined as

$$
\operatorname{bmo}\left(\mathbb{R}^{n}\right)=\left\{f \in L_{\text {loc }}^{1}\left(\mathbb{R}^{n}\right): \mathcal{N} f \in L^{\infty}\left(\mathbb{R}^{n}\right)\right\}
$$

where

$$
\mathcal{N} f(x)=\sup _{B \in \mathcal{B}_{1}(x)} \frac{1}{V(B)} \int_{B}\left|f(y)-f_{B}\right| d y+\frac{1}{V\left(B_{1}(x)\right)} \int_{B_{1}(x)}|f(y)| d y
$$

with (in place of (1.8))

$$
\mathcal{B}_{1}(x)=\left\{B_{r}(x): 0<r \leq 1\right\}
$$

As shown in $[G]$, the spaces $\mathfrak{h}^{1}\left(\mathbb{R}^{n}\right)$ and $\operatorname{bmo}\left(\mathbb{R}^{n}\right)$ are invariant under the action of multiplication $(f \mapsto a f)$ by nice functions, and more generally invariant under the action of pseudodifferential operators $p(x, D) \in O P S_{1,0}^{0}\left(\mathbb{R}^{n}\right)$, where we recall

$$
\begin{gathered}
p(x, D) f(x)=(2 \pi)^{-n} \iint p(x, \xi) f(y) e^{i(x-y) \cdot \xi} d y d \xi \\
p(x, \xi) \in S_{1,0}^{m}\left(\mathbb{R}^{n}\right) \Leftrightarrow\left|D_{x}^{\beta} D_{\xi}^{\alpha} p(x, \xi)\right| \leq C_{\alpha \beta}(1+|\xi|)^{m-|\alpha|},
\end{gathered}
$$

and then say $p(x, D) \in O P S_{1,0}^{m}\left(\mathbb{R}^{n}\right)$. Invariance under a class of diffeomorphisms on $\mathbb{R}^{n}$ is also established, allowing one to define $\mathfrak{h}^{1}(M)$ and $\operatorname{bmo}(M)$ whenever $M$ is a smooth, compact manifold. An alternative approach to $\mathfrak{h}^{1}(M)$ for such $M$ had been given in [Str1].

In $[\mathrm{CKS}]$, a theory of local Hardy spaces was developed on smoothly bounded domains, and applied to some elliptic boundary problems.

In another direction, [CW1]-[CW2] have studied $H^{1}(X)$ and $\mathrm{BMO}(X)$ when $X$ is a space of "homogeneous type," a metric space (or more generally a quasi-metric space) with a measure satisfying a doubling condition.

Another class of spaces on which many people do analysis is the class of symmetric spaces of noncompact type, such as $n$-dimensional hyperbolic space $\mathcal{H}^{n}$. Despite the fact that these are homogeneous spaces, they are not spaces of "homogeneous type," since balls of large radius grow too rapidly in volume, a fact that influences analysis on these spaces in many ways. In the course of studying some Fourier integral operators on $\mathcal{H}^{n}$ (and other noncompact symmetric spaces of real rank 1) [I] defines BMO and develops basic properties. Somewhat parallel to (1.6)-(1.8), [I] takes

$$
\operatorname{BMO}\left(\mathcal{H}^{n}\right)=\left\{f \in L_{\text {loc }}^{1}\left(\mathcal{H}^{n}\right): f^{\#} \in L^{\infty}\left(\mathcal{H}^{n}\right)\right\}
$$

where

$$
f^{\#}(x)=\sup _{B \in \mathcal{B}_{1}(x)} \frac{1}{V(B)} \int_{B}\left|f(y)-f_{B}\right| d V(y)
$$


but, in contrast to $(1.8), \mathcal{B}_{1}(x)$ is as in (1.15). This contrast makes it problematic to produce a unified theory of the spaces $H^{1}(M)$ and $\operatorname{BMO}(M)$ for a class of manifolds including both $M=\mathbb{R}^{n}$ and $M=\mathcal{H}^{n}$.

Our goal in this paper is to produce a unified theory of the "local" spaces $\mathfrak{h}^{1}(M)$ and $\operatorname{bmo}(M)$, whenever $M$ is a complete Riemannian manifold with bounded geometry. We define "bounded geometry" as follows. First we assume there exists $R_{0} \in(0, \infty)$ such that for each $p \in M$, the exponential map

$$
\operatorname{Exp}_{p}: T_{p} M \longrightarrow M
$$

has the property

$$
\operatorname{Exp}_{p}: B_{R_{0}}(0) \longrightarrow B_{R_{0}}(p) \text { diffeomorphically, }
$$

where $B_{r}(p)=\{x \in M: d(x, p)<r\}, d(x, p)$ denoting the distance from $x$ to $p$. Furthermore, the pull-back of the metric tensor from $B_{R_{0}}(p) \subset M$ to $B_{R_{0}}(0) \subset$ $T_{p} M$, identified with $B_{R_{0}}(0) \subset \mathbb{R}^{n}(n=\operatorname{dim} M)$, uniquely up to an element of $O(n)$, furnishes a collection of $n \times n$ matrices $G_{p}(x)=\left(g_{j k}^{p}(x)\right)$ satisfying

$$
\left\{G_{p}: p \in M\right\} \text { is bounded in } C^{\infty}\left(B_{R_{0}}(0), \operatorname{End}\left(\mathbb{R}^{n}\right)\right) \text {. }
$$

We also require that

$$
\xi \cdot G_{p}(x) \xi \geq \frac{1}{2}|\xi|^{2}, \quad \forall p \in M, x \in B_{R_{0}}(0), \xi \in \mathbb{R}^{n},
$$

and that

$$
B_{R_{0}}(p) \text { is geodesically convex, } \forall p \in M \text {. }
$$

Such is a complete Riemannian manifold with bounded geometry. Given this, we find it convenient to multiply the metric tensor of $M$ by a constant, if necessary, so we can say the properties above hold with

$$
R_{0}=4
$$

Having (1.20)-(1.24), we can pick $p_{k} \in M, k \in \mathbb{Z}^{+}$, such that

$$
\left\{B_{1 / 2}\left(p_{k}\right): k \in \mathbb{Z}^{+}\right\} \text {covers } M
$$

while, for some $K=K(M)<\infty$,

$$
\forall p \in M \text {, at most } K \text { balls } B_{2}\left(p_{k}\right) \text { contain } p \text {. }
$$

We can then form a partition of unity $\sum_{k} \varphi_{k}=1$ such that

$$
\operatorname{supp} \varphi_{k} \subset B_{1}\left(p_{k}\right), \quad \varphi_{k} \circ \operatorname{Exp}_{p_{k}} \text { is bounded in } C_{0}^{\infty}\left(B_{1}(0)\right) \text {. }
$$


We call such $\left\{\varphi_{k}: k \in \mathbb{Z}^{+}\right\}$a tame partition of unity, and the collection $\left\{B_{1}\left(p_{k}\right)\right.$ : $\left.k \in \mathbb{Z}^{+}\right\}$a tame cover of $M$.

The structure of the rest of this paper is as follows. In Sections 2 and 3, respectively, we define $\mathfrak{h}^{1}(M)$ and $\operatorname{bmo}(M)$, when $M$ has bounded geometry, and establish some basic properties, starting with showing that

$$
f \in \mathfrak{h}^{1}(M), g \in \operatorname{bmo}(M) \Longrightarrow a f \in \mathfrak{h}^{1}(M), a g \in \operatorname{bmo}(M),
$$

for a class of functions a containing $L^{\infty}(M) \cap \operatorname{Lip}(M)$. Having these results, we then show that

$$
\begin{aligned}
\|f\|_{\mathfrak{h}^{1}(M)} & \approx \sum_{k}\left\|\left(\varphi_{k} f\right) \circ \operatorname{Exp}_{p_{k}}\right\|_{\mathfrak{h}^{1}\left(\mathbb{R}^{n}\right)}, \\
\|g\|_{\text {bmo }(M)} & \approx \sup _{k}\left\|\left(\varphi_{k} g\right) \circ \operatorname{Exp}_{p_{k}}\right\|_{\text {bmo }\left(\mathbb{R}^{n}\right)},
\end{aligned}
$$

where $\left\{\varphi_{k}: k \in \mathbb{Z}^{+}\right\}$is a tame partition of unity, as in (1.27). The spaces $\mathfrak{h}^{1}\left(\mathbb{R}^{n}\right)$ and $\operatorname{bmo}\left(\mathbb{R}^{n}\right)$ are as in (1.10)-(1.15). This enables us to make use of the results of $[\mathrm{G}]$ (and, by extension, those of $[\mathrm{FS}]$ ). Doing this, we show in $\S 4$ that

$$
\mathfrak{h}^{1}(M)^{\prime}=\operatorname{bmo}(M)
$$

whenever $M$ has bounded geometry, extending (1.12).

In $\S 5$ we discuss the atomic theory of $\mathfrak{h}^{1}(M)$. We relate this to the "ionic theory," developed in the context of Lipschitz surfaces in [MT]

In $\S 6$ we show that when $M$ has bounded geometry,

$$
(\lambda I-\Delta)^{i t}: \operatorname{bmo}(M) \longrightarrow \operatorname{bmo}(M)
$$

with an exponential bound, provided $\lambda \geq \lambda_{0}(M)$ is sufficiently large. We also get such bounds on $\mathfrak{h}^{1}(M)$ and on $L^{p}(M)$, for $p \in(1, \infty)$. We show that the operators in (1.31) belong to a class of pseudodifferential operators on $M$ denoted $\Psi_{W}^{0}(M)$, given $W<\sqrt{\lambda}$, where $\Psi_{W}^{m}(M)$ consists of operators of the form

$$
P=P^{\#}+P^{b}
$$

of the following nature. The Schwartz kernel of $P^{\#}$ is supported near the diagonal in $M \times M$ and in local exponential coordinates centered at $p \in M, P^{\#}$, acting on functions supported in $B_{4}(p)$ (identified with a ball in $\mathbb{R}^{n}$ ) belongs to the class $O P S_{1,0}^{m}\left(\mathbb{R}^{n}\right)$ of classical pseudodifferential operators on $\mathbb{R}^{n}$, with bounds independent of $p$. Meanwhile $P^{b}$ has integral kernel $K^{b}(x, y)$, satisfying estimates

$$
\left|K^{b}(x, y)\right| \leq C_{k}(1+d(x, y))^{-k} e^{-W d(x, y)}, \quad \forall k \in \mathbb{Z}^{+},
$$

plus such estimates on all its derivatives. Generalizing (1.31), we get

$$
P \in \Psi_{W}^{0}(M), W \geq K_{0} \Longrightarrow P: \operatorname{bmo}(M) \rightarrow \operatorname{bmo}(M),
$$


and also bounds on $\mathfrak{h}^{1}(M)$ and on $L^{p}(M), p \in(1, \infty)$. Here $K_{0}$ is a geometrical constant; cf. (6.16). We also show that

$$
P_{1} \in \Psi_{W}^{m_{1}}(M), P_{2} \in \Psi_{W+K_{1}}^{m_{2}}(M), K_{1}>K_{0} \Longrightarrow P_{1} P_{2}, P_{2} P_{1} \in \Psi_{W}^{m_{1}+m_{2}}(M) .
$$

These results will be of further use later on.

Another fundamental circle of results in [FS] involved interpolation, showing for example that if $T: L^{2}\left(\mathbb{R}^{n}\right) \rightarrow L^{2}\left(\mathbb{R}^{n}\right)$ and also $T: L^{\infty}\left(\mathbb{R}^{n}\right) \rightarrow \operatorname{BMO}\left(\mathbb{R}^{n}\right)$, then $T: L^{p}\left(\mathbb{R}^{n}\right) \rightarrow L^{p}\left(\mathbb{R}^{n}\right)$ for each $p \in(2, \infty)$. A key ingredient was an $L^{p}$ estimate on $f$ in terms of $f^{\#}$, defined by (1.7). In $\S \S 7-9$ of this paper we establish analogous results for manifolds with bounded geometry, involving bmo $(M)$. In $\S 7$ we obtain local estimates for $\|f\|_{L^{p}}$ on a cube $Q$, in terms of $\left.f^{\#}\right|_{Q}$ and $\|f\|_{L^{1}(Q)}$. We apply this in $\S 8$ to obtain estimates of the form

$$
\|f\|_{L^{p}(M)} \leq C_{p}\|\mathcal{N} f\|_{L^{p}(M)}, \quad 1<p<\infty,
$$

when $M$ is a manifold with bounded geometry and $\mathcal{N} f$ is defined as in (3.4). The interpolation result is then established in $\S 9$.

Section 10 introduces $L^{p}$, Hardy, and bmo-Sobolev spaces on $M$ and discusses some basic properties. We begin with definitions of

$$
H^{k, p}(M), \quad \mathfrak{h}^{k, 1}(M), \quad \mathfrak{h}^{k, \infty}(M),
$$

when $k \in \mathbb{N}$, and then define

$$
H^{s, p}(M), \quad \mathfrak{h}^{s, 1}(M), \quad \mathfrak{h}^{s, \infty}(M),
$$

for $s \in \mathbb{R}$. Our first order of business is to show the definitions of (1.38) are equivalent to those of (1.37) when $s=k \in \mathbb{N}$. Results on $\Psi_{W}^{m}(M)$ from $\S 6$ are useful here, together with the fact that

$$
(\lambda I-\Delta)^{-m / 2} \in \Psi_{W}^{-m}(M)
$$

when $m \in \mathbb{R}$ and $\lambda>\sqrt{W}$. One result of $\S 10$ is that

$$
P \in \Psi_{W}^{m}(M), W \geq K_{0} \Longrightarrow P: \mathfrak{h}^{s, \infty}(M) \rightarrow \mathfrak{h}^{s-m, \infty}(M)
$$

and corresponding results for the action of such $P$ on $\mathfrak{h}^{s, 1}(M)$ and on $H^{s, p}(M)$ for $p \in(1, \infty)$.

In $§ 11$ we establish further interpolation results, of the following nature. Given

$$
R: L^{2}(M) \rightarrow L^{2}(M), \quad R: L^{1}(M) \rightarrow \mathfrak{h}^{s, \infty}(M),
$$

we have, for $\theta \in(0,1)$,

$$
R: L^{p}(M) \longrightarrow H^{(1-\theta) s, p^{\prime}}(M), \quad p=\frac{2}{2-\theta}, p^{\prime}=\frac{2}{\theta},
$$


together with associated operator norm bounds. Such results are applicable to estimates on

$$
S(t)=\frac{\sin t \sqrt{-\Delta}}{\sqrt{-\Delta}},
$$

for which one has bounds

$$
\|S(t) f\|_{H^{1,2}} \leq A_{1}(t)\|f\|_{L^{2}}, \quad\|S(t) f\|_{\mathfrak{h}^{-(n-1) / 2, \infty}} \leq A_{0}(t)\|f\|_{L^{1}}
$$

at least under certain additional geometrical hypotheses on $M$. We can apply (1.41)-(1.42) to $R(t)=(\lambda I-\Delta)^{1 / 2} S(t)$, with $\lambda>K_{0}^{2}$. The resulting operator estimates on $S(t)$ (known as dispersive estimates) have potential application to nonlinear wave equations on various classes of Riemannian manifolds, such as hyperbolic space. These matters will be taken up elsewhere.

In Appendix A we study the space $\operatorname{vmo}(M)$, which is the closure in $\operatorname{bmo}(M)$ of $C_{0}^{\infty}(M)$, in analogy with $\operatorname{VMO}\left(\mathbb{R}^{n}\right)$, introduced in [Sar] as the closure in $\operatorname{BMO}\left(\mathbb{R}^{n}\right)$ of $C_{0}^{\infty}\left(\mathbb{R}^{n}\right)$. As shown in $[$ Sar]

$$
\operatorname{VMO}\left(\mathbb{R}^{n}\right)^{\prime}=H^{1}\left(\mathbb{R}^{n}\right) .
$$

We show that, when $M$ has bounded geometry,

$$
\operatorname{vmo}(M)^{\prime}=\mathfrak{h}^{1}(M) .
$$

We also show that, under the hypotheses of (1.34),

$$
P: \operatorname{vmo}(M) \longrightarrow \operatorname{vmo}(M) \text {. }
$$

In Appendix B, we introduce another class of pseudodifferential operators:

$$
\widetilde{\Psi}_{W}^{m}(M) \subset \Psi_{W}^{m}(M)
$$

It is of some interest that functions of the Laplace operator, such as discussed in $\S 6$ and $\S 10$, actually belong to this smaller space, particularly in view of the fact that elements of $\widetilde{\Psi}_{W}^{0}(M)$ have sharper $L^{p}$-operator estimates. These results tie in closely with results of [T3].

In Appendix C, we point out some special geometrical and analytic properties of symmetric spaces of noncompact type. We extend $L^{p}$-Sobolev space bounds on operators of the form (1.39) to the result that

$$
(-\Delta)^{m / 2}: H^{s, p}(M) \longrightarrow H^{s-m, p}(M)
$$

for $m, s \in \mathbb{R}, p \in(1, \infty)$, in the special case that $M$ is a symmetric space of noncompact type. We indicate how this leads to an extension of the dispersive 
estimates on operators of the form (1.43) described in $\S 11$ to dispersive estimates on $e^{i t \sqrt{-\Delta}}$, in this setting.

REMARK. We call attention to the recent work [CMM], developing a theory of $H^{1}$ and $\mathrm{BMO}$ for certain nondoubling measured metric measure spaces. This paper takes the approach to $H^{1}$ and BMO of [I] and extends its scope considerably. Our paper, emphasizing the "local" spaces $\mathfrak{h}^{1}$ and bmo, is to some degree complementary to $[\mathrm{CMM}]$, though these two papers deal with a number of common themes.

\section{The space $\mathfrak{h}^{1}(M)$}

We take $M$ to be a complete Riemannian manifold, of dimension $n$, with bounded geometry, as defined in the Introduction. As described there, we scale the metric tensor (if necessary) to arrange that the properties (1.20)-(1.24) hold. Given $f \in$ $L_{\text {loc }}^{1}(M)$, we define the following maximal function:

$$
\mathcal{G}^{b} f(x)=\sup _{0<r \leq 1} \mathcal{G}_{r} f(x),
$$

where

$$
\mathcal{G}_{r} f(x)=\sup \left\{\left|\int \varphi(y) f(y) d V(y)\right|: \varphi \in \mathcal{F}\left(B_{r}(x)\right)\right\}
$$

with

$$
\mathcal{F}\left(B_{r}(x)\right)=\left\{\varphi \in C_{0}^{1}\left(B_{r}(x)\right):\|\varphi\|_{\text {Lip }} \leq \frac{1}{r^{n+1}}\right\}
$$

We then set

$$
\mathfrak{h}^{1}(M)=\left\{f \in L_{\text {loc }}^{1}(M): \mathcal{G}^{b} f \in L^{1}(M)\right\}
$$

with norm

$$
\|f\|_{\mathfrak{h}^{1}}=\left\|\mathcal{G}^{b} f\right\|_{L^{1}} .
$$

Remark. One could replace $C_{0}^{1}\left(B_{r}(x)\right)$ by $\left\{\varphi \in \operatorname{Lip}(M): \operatorname{supp} \varphi \subset B_{r}(x)\right\}$ and get the same result.

A comparison with (1.10)-(1.11) shows that when $M=\mathbb{R}^{n}$, the space $\mathfrak{h}^{1}(M)$ defined above coincides with the space $\mathfrak{h}^{1}\left(\mathbb{R}^{n}\right)$ defined in the Introduction.

It is convenient to know that $\mathfrak{h}^{1}(M)$ is a module over $\operatorname{Lip}(M) \cap L^{\infty}(M)$. In fact, a more precise result holds. Let $\sigma$ be a modulus of continuity, and say

$$
a \in C^{\sigma}(M) \Longleftrightarrow|a(x)-a(y)| \leq L \sigma(d(x, y)), \text { for } d(x, y) \leq 1,
$$

for some $L \in[0, \infty)$. Define $\|a\|_{C^{\sigma}}$ to be the smallest $L$ for which (2.6) holds (this is a seminorm). We then have the following result. 
Proposition 2.1. Let $\sigma$ be a modulus of continuity satisfying the Dini condition

$$
D(\sigma)=\int_{0}^{1} \frac{\sigma(r)}{r} d r<\infty
$$

We also assume $\sigma(r) / r$ is monotonically decreasing on $(0,1]$ (or constant). Then

$$
a \in L^{\infty}(M) \cap C^{\sigma}(M), f \in \mathfrak{h}^{1}(M) \Longrightarrow a f \in \mathfrak{h}^{1}(M)
$$

The proof is quite similar to that for the Euclidean case, but for the sake of completeness we give the details.

Take $a$ and $f$ as in (2.8). To estimate $\mathcal{G}^{b}(a f)(x)$, we compare $\mathcal{G}_{r}(a f)(x)$ with $a(x) \mathcal{G}_{r} f(x)$. Take $\varphi \in \mathcal{F}\left(B_{r}(x)\right)$, and note that

$$
\varphi \in \mathcal{F}\left(B_{r}(x)\right) \Longrightarrow\|\varphi\|_{L^{\infty}} \leq \frac{1}{r^{n}}
$$

Hence

$$
\begin{aligned}
& \left|\int_{B_{r}(x)} \varphi(y)[a(y) f(y)-a(x) f(y)] d V(y)\right| \\
& \quad \leq \sup _{d(x, y) \leq r}|a(x)-a(y)| \int|\varphi(y)| \cdot|f(y)| d V(y) \\
& \quad \leq\|a\|_{C^{\sigma}} \frac{\sigma(r)}{r^{n}} \int_{B_{r}(x)}|f(y)| d V(y) .
\end{aligned}
$$

That is to say,

$$
\left|\mathcal{G}_{r}(a f)(x)-a(x) \mathcal{G}_{r} f(x)\right| \leq\|a\|_{C^{\sigma}} \frac{\sigma(r)}{r^{n}} \int_{B_{r}(x)}|f| d V .
$$

Hence, by (2.1),

$$
\mathcal{G}^{b}(a f)(x) \leq|a(x)| \mathcal{G}^{b} f(x)+\|a\|_{C^{\sigma}} \mathcal{R}^{\sigma} f(x),
$$

where

$$
\begin{aligned}
\mathcal{R}^{\sigma} f(x) & =\sup _{0<r \leq 1} \frac{\sigma(r)}{r^{n}} \int_{B_{r}(x)}|f| d V \\
& \leq \int_{B_{1}(x)} \frac{\sigma(d(x, y))}{d(x, y)^{n}}|f(y)| d V(y) \\
& =\int_{M} K^{\sigma}(x, y)|f(y)| d V(y),
\end{aligned}
$$


where the last identity defines $K^{\sigma}(x, y)$. Note that $K^{\sigma}(x, y) \geq 0$ and

$$
\begin{aligned}
\int_{M} K^{\sigma}(x, y) d V(x) & =\int_{B_{1}(y)} \frac{\sigma(d(x, y))}{d(x, y)^{n}} d V(x) \\
& \leq C \int_{0}^{1} \frac{\sigma(r)}{r} d r
\end{aligned}
$$

with $C<\infty$ depending on the geometrical bounds for $M$. Consequently

$$
\left\|\mathcal{R}^{\sigma} f\right\|_{L^{1}} \leq C D(\sigma)\|f\|_{L^{1}}
$$

and we have the desired estimate for (2.12), yielding

$$
\left\|\mathcal{G}^{b}(a f)\right\|_{L^{1}} \leq\|a\|_{L^{\infty}}\left\|\mathcal{G}^{b} f\right\|_{L^{1}}+C D(\sigma)\|a\|_{C^{\sigma}}\|f\|_{L^{1}}
$$

This proves Proposition 2.1.

Using Proposition 2.1, we can establish the following.

Proposition 2.2. Let $\left\{\varphi_{k}: k \in \mathbb{Z}^{+}\right\}$be a tame partition of unity. Given $f \in$ $L_{\text {loc }}^{1}(M)$, we have

$$
f \in \mathfrak{h}^{1}(M) \Longleftrightarrow \sum_{k}\left\|\varphi_{k} f\right\|_{\mathfrak{h}^{1}}<\infty
$$

and

$$
\|f\|_{\mathfrak{h}^{1}} \approx \sum_{k}\left\|\varphi_{k} f\right\|_{\mathfrak{h}^{1}}
$$

Proof. The inequality $\|f\|_{\mathfrak{h}^{1}} \leq \sum_{k}\left\|\varphi_{k} f\right\|_{\mathfrak{h}^{1}}$ is elementary. For the converse estimate in (2.18), we argue as follows. We can partition the set $\left\{p_{k}: k \in \mathbb{Z}^{+}\right\}$ mentioned in (1.25)-(1.27) into $K_{1}=K_{1}(M)$ subsets $\mathcal{S}_{1}, \ldots \mathcal{S}_{K_{1}}$ such that

$$
p_{j}, p_{k} \in \mathcal{S}_{\nu}, j \neq k \Longrightarrow d\left(p_{j}, p_{k}\right) \geq 20
$$

For convenience, if $p_{k} \in \mathcal{S}_{\nu}$, we also write $k \in \mathcal{S}_{\nu}$. For each $\nu \in\left\{1, \ldots, K_{1}\right\}$, set

$$
T_{\nu} f=\sum_{k \in \mathcal{S}_{\nu}} \varphi_{k} f
$$

We apply Proposition 2.1 to $a=a_{\nu}=\sum_{k \in \mathcal{S}_{\nu}} \varphi_{k}$ and deduce that

$$
T_{\nu}: \mathfrak{h}^{1}(M) \rightarrow \mathfrak{h}^{1}(M), \quad\left\|T_{\nu} f\right\|_{\mathfrak{h}^{1}} \leq C\|f\|_{\mathfrak{h}^{1}}, \quad 1 \leq \nu \leq K_{1}
$$


On the other hand, by the degree of disjointness of the supports of $\left\{\varphi_{k}: k \in \mathcal{S}_{\nu}\right\}$, a direct check of the definitions gives

$$
\mathcal{G}^{b}\left(\sum_{k \in \mathcal{S}_{\nu}} \varphi_{k} f\right)=\sum_{k \in \mathcal{S}_{\nu}} \mathcal{G}^{b}\left(\varphi_{k} f\right),
$$

for each $\nu$, and (again by disjointness)

$$
\sum_{k \in \mathcal{S}_{\nu}}\left\|\mathcal{G}^{b}\left(\varphi_{k} f\right)\right\|_{L^{1}}=\left\|\mathcal{G}^{b} T_{\nu} f\right\|_{L^{1}}
$$

i.e.,

$$
\sum_{k \in \mathcal{S}_{\nu}}\left\|\varphi_{k} f\right\|_{\mathfrak{h}^{1}}=\left\|T_{\nu} f\right\|_{\mathfrak{h}^{1}}
$$

Summing over $\nu \in\left\{1, \ldots, K_{1}\right\}$ then gives

$$
\sum_{k}\left\|\varphi_{k} f\right\|_{\mathfrak{h}^{1}} \leq C K_{1}\|f\|_{\mathfrak{h}^{1}}
$$

proving (2.18).

Proposition 2.2 combines nicely with the following elementary result.

Proposition 2.3. We have, uniformly in $k \in \mathbb{Z}^{+}$,

$$
\left\|\varphi_{k} f\right\|_{\mathfrak{h}^{1}(M)} \approx\left\|\left(\varphi_{k} f\right) \circ \operatorname{Exp}_{p_{k}}\right\|_{\mathfrak{h}^{1}\left(\mathbb{R}^{n}\right)}
$$

We recall that there is an isometric isomorphism of the $n$-dimensional inner product space $T_{p} M$ with $\mathbb{R}^{n}$, determined uniquely up to the action of $O(n)$.

Corollary 2.4. In the setting of Proposition 2.2,

$$
\|f\|_{\mathfrak{h}^{1}(M)} \approx \sum_{k}\left\|\left(\varphi_{k} f\right) \circ \operatorname{Exp}_{p_{k}}\right\|_{\mathfrak{h}^{1}\left(\mathbb{R}^{n}\right)}
$$

The following result is occasionally useful.

Proposition 2.5. The space $C_{0}^{\infty}(M)$ is dense in $\mathfrak{h}^{1}(M)$.

Proof. Take $f \in \mathfrak{h}^{1}(M)$. Via Proposition 2.2, it suffices to approximate each term $\varphi_{k} f$ in $\mathfrak{h}^{1}$-norm by an element of $C_{0}^{\infty}\left(B_{2}\left(p_{k}\right)\right) \approx C_{0}^{\infty}\left(B_{2}(0)\right)$. This reduces matters to treating the case $M=\mathbb{R}^{n}$. In this case, the result is proven in [G], p. 35 .

We record some other useful facts. First, the Lebesgue density theorem immediately gives

$$
|f(x)| \leq C \mathcal{G}^{b} f(x)
$$

a.e. on $M$, for each $f \in L_{\text {loc }}^{1}(M)$, with $C=C(M)<\infty$. We also have the following: 
Proposition 2.6. Assume $f$ is a locally finite Borel measure on $M$. Define $\mathcal{G}^{b} f(x)$ as the natural variant of (2.1)-(2.1). If $\mathcal{G}^{b} f \in L^{1}(M)$, then $f \in L_{\text {loc }}^{1}(M)$, hence $f \in \mathfrak{h}^{1}(M)$.

Proof. Let $J_{\varepsilon}$ be a mollifier, with integral kernel supported in $\{(x, y): d(x, y) \leq \varepsilon\}$. Then $f_{\varepsilon}=J_{\varepsilon} f \in C^{\infty}(M)$ and $f_{\varepsilon} \rightarrow f$ weak* in $\mathcal{M}_{\text {loc }}(M)$. Since $\left\langle\varphi, f_{\varepsilon}\right\rangle=\left\langle J_{\varepsilon}^{*} \varphi, f\right\rangle$, we have, for $\varepsilon \in(0,1]$,

$$
\begin{aligned}
\mathcal{G}^{b} f_{\varepsilon}(x) & \leq C \sup _{0<r \leq 1+\varepsilon} \mathcal{G}_{r} f(x) \\
& \leq C \mathcal{G}^{b} f(x)+C_{1}(x),
\end{aligned}
$$

where $C_{1}(x)$ is the total variation of $f$ on $B_{2}(x)$, a locally bounded function. We can apply $(2.28)$ to $f_{\varepsilon}$ and get

$$
\left|f_{\varepsilon}(x)\right| \leq C \mathcal{G}^{b} f(x)+C_{1}(x)
$$

again with $C<\infty, C_{1}(x)$ locally bounded, independent of $\varepsilon \in(0,1]$. The uniform estimate (2.30) implies $f \in L_{\text {loc }}^{1}(M)$, so we are in the setting of the definition of $\mathfrak{h}^{1}(M)$ given at the beginning of this section.

\section{The space $\operatorname{bmo}(M)$}

As usual, $M$ is a complete Riemannian manifold, of dimension $n$, with bounded geometry, and with the properties (1.20)-(1.24). We set up the following maximal functions. Given $f \in L_{\text {loc }}^{1}(M)$, let

$$
f^{\#}(x)=\sup _{B \in \mathcal{B}(x)} \frac{1}{V(B)} \int_{B}\left|f-f_{B}\right| d V
$$

where

$$
f_{B}=\frac{1}{V(B)} \int_{B} f d V
$$

and

$$
\mathcal{B}(x)=\left\{B_{r}(x): 0<r \leq 1\right\}
$$

Then define

$$
\mathcal{N} f(x)=f^{\#}(x)+\mathcal{N}_{0} f(x), \quad \mathcal{N}_{0} f(x)=\frac{1}{V\left(B_{1}(x)\right)} \int_{B_{1}(x)}|f| d V .
$$


We set

$$
\operatorname{bmo}(M)=\left\{f \in L_{\text {loc }}^{1}(M): \mathcal{N} f \in L^{\infty}(M)\right\}
$$

with norm

$$
\|f\|_{\text {bmo }}=\|\mathcal{N} f\|_{L^{\infty}}
$$

In case $M=\mathbb{R}^{n}$, the definition of $\operatorname{bmo}(M)$ given here is clearly equivalent to that of $\operatorname{bmo}\left(\mathbb{R}^{n}\right)$ given in the Introduction.

It is useful to make note of some equivalent norms. For example, in place of $f \#$, consider

$$
f^{s}(x)=\sup _{B \in \mathcal{B}(x)} \inf _{c_{B} \in \mathbb{C}} \frac{1}{V(B)} \int_{B}\left|f-c_{B}\right| d V
$$

Given $B \in \mathcal{B}(x)$ and taking $c_{B}$ to realize this infimum, we have

$$
\begin{aligned}
\left|f_{B}-c_{B}\right| & =\left|\frac{1}{V(B)} \int_{B}\left(f-c_{B}\right) d V\right| \\
& \leq \frac{1}{V(B)} \int_{B}\left|f-c_{B}\right| d V \\
& \leq f^{s}(x)
\end{aligned}
$$

and hence

$$
\frac{1}{V(B)} \int_{B}\left|f-f_{B}\right| d V \leq \frac{1}{V(B)} \int_{B}\left(\left|f-c_{B}\right|+\left|f_{B}-c_{B}\right|\right) d V \leq 2 f^{s}(x) .
$$

Consequently,

$$
f^{s}(x) \leq f^{\#}(x) \leq 2 f^{s}(x)
$$

It is also useful to note that one can fix $a, b, c \in(0, \infty)$, with $a<b$, and replace $\mathcal{B}(x)$ by

$$
\widetilde{\mathcal{B}}(x)=\left\{Q_{r}^{\alpha}(x): 0<r \leq 1, \alpha \in \mathcal{A}\right\}
$$

where $Q_{r}^{\alpha}(x)$ is a family of measurable sets with the property that for each $r \in(0,1]$,

$$
\begin{aligned}
V\left(Q_{r}^{\alpha}(x)\right) \geq c V\left(B_{r}(x)\right), & Q_{r}^{\alpha}(x) \subset B_{b r}(x), \quad \text { for all } \alpha, \text { and } \\
& B_{a r}(x) \subset Q_{r}^{\alpha}(x), \quad \text { for some } \alpha .
\end{aligned}
$$


One gets functions comparable in size in (3.7) and hence also in (3.1). In connection with this, we recall that the original treatments in [JN] and [FS] used cubes containing $x$ in place of balls centered at $x$. One consequence of this observation is that the John-Nirenberg estimate, proven in [JN] for functions defined on a cube in $\mathbb{R}^{n}$, is applicable in our current situation. We have, for each ball $B \subset M$ of radius $\leq 1$

$$
\frac{1}{V(B)} \int_{B} e^{\alpha\left|f-f_{B}\right|} d V \leq \gamma
$$

with

$$
\alpha=\frac{\beta}{\|f\|_{\mathrm{bmo}}}, \quad \beta, \gamma \text { constants }
$$

Cf. $\left(3^{\prime}\right)$ of $[\mathrm{JN}]$.

We next aim to show that

$$
a \in \operatorname{Lip}(M) \cap L^{\infty}(M), f \in \operatorname{bmo}(M) \Longrightarrow a f \in \operatorname{bmo}(M) .
$$

In fact, we will obtain a much more precise result, which can be compared with Proposition 2.1. To begin, note that

$$
\mathcal{N}_{0}(a f)(x) \leq\|a\|_{L \infty} \mathcal{N}_{0}(f)(x)
$$

so it suffices to estimate $(a f)^{s}(x)$. For $B \in \mathcal{B}(x)$, we take $c_{B}=a(x) f_{B}$. Then we have

$$
\begin{aligned}
\frac{1}{V(B)} & \int_{B}\left|a(y) f(y)-a(x) f_{B}\right| d V(y) \\
& \leq \frac{1}{V(B)} \int_{B}|a| \cdot\left|f-f_{B}\right| d V+\frac{1}{V(B)} \int_{B}|a(y)-a(x)| \cdot\left|f_{B}\right| d V(y) \\
& \leq\|a\|_{L^{\infty}} f^{\#}(x)+\left(\sup _{y \in B}|a(x)-a(y)|\right)\left|f_{B}\right| .
\end{aligned}
$$

Let us assume that $a \in C^{\sigma}(M)$, defined in (2.6). The last term in (3.17) is bounded by

$$
\|a\|_{C^{\sigma}} \sigma(r)\left|f_{B}\right|, \quad \text { if } B=B_{r}(x) .
$$

We use the John-Nirenberg estimate (3.13)-(3.14) to estimate $\left|f_{B}\right|$. With $\alpha$ as in (3.14), $B=B_{r}(x), 0<r \leq 1$,

$$
\begin{aligned}
e^{\alpha\left|f_{B}\right| / 2} & =\frac{1}{V(B)} \int_{B} e^{\alpha\left|f_{B}\right| / 2} d V \\
& =\frac{1}{V(B)} \int_{B} e^{\alpha\left|f-\left(f-f_{B}\right)\right| / 2} d V \\
& \leq \frac{1}{V(B)} \int_{B_{1}} e^{\alpha|f|} d V+\frac{1}{V(B)} \int_{B} e^{\alpha\left|f-f_{B}\right|} d V
\end{aligned}
$$


where $B_{1}=B_{1}(x)$. The estimate (3.13) applies to the second term on the last line of (3.19). To estimate the first term on that line, we have

$$
\begin{aligned}
\int_{B_{1}} e^{\alpha|f|} d V & =\int_{B_{1}} e^{\alpha\left|f-f_{B_{1}}+f_{B_{1}}\right|} d V \\
& \leq e^{\alpha\left|f_{B_{1}}\right|} \int_{B_{1}} e^{\alpha\left|f-f_{B_{1}}\right|} d V \\
& \leq \gamma V\left(B_{1}\right) e^{\alpha\left|f_{B_{1}}\right|} \\
& \leq \gamma V\left(B_{1}\right) e^{\beta}
\end{aligned}
$$

the penultimate inequality by (3.13) and the last inequality because

$$
\left|f_{B_{1}}\right| \leq V\left(B_{1}\right)^{-1} \int_{B_{1}}|f| d V \leq\|f\|_{\text {bmo }}
$$

Using this in (3.19) we get (for some constant $\gamma_{1}$ )

$$
e^{\alpha\left|f_{B}\right| / 2} \leq \frac{\gamma_{1}}{V(B)}, \text { hence }\left|f_{B}\right| \leq \frac{2}{\alpha} \log \frac{\gamma_{1}}{V(B)}
$$

Recalling that our goal is to estimate (3.18), we have

$$
\begin{aligned}
\sigma(r)\left|f_{B}\right| & \leq \frac{2}{\alpha} \sigma(r) \log \frac{\gamma_{2}}{r^{n}} \\
& =\frac{2 n}{\beta}\|f\|_{\mathrm{bmo}} \sigma(r) \log \frac{\gamma_{3}}{r}
\end{aligned}
$$

We have the following sharpening of (3.15).

Proposition 3.1. If $a \in L^{\infty}(M) \cap C^{\sigma}(M)$ with

$$
\sigma(r)=\left(\log \frac{1}{r}\right)^{-1}, \quad 0<r \leq \frac{1}{2}
$$

then

$$
f \in \operatorname{bmo}(M) \Longrightarrow a f \in \operatorname{bmo}(M)
$$

REMARK. Note that the Dini condition (2.7) just barely fails for $\sigma(r)$ given by (3.23). We discuss this further after establishing $\mathfrak{h}^{1}$-bmo duality in $\S 4$.

We can use Proposition 3.1 to establish the following counterpart to Proposition 2.2 . 
Proposition 3.2. Let $\left\{\varphi_{k}: k \in \mathbb{Z}^{+}\right\}$be a tame partition of unity. Given $f \in$ $L_{\text {loc }}^{1}(M)$, we have

$$
f \in \operatorname{bmo}(M) \Longleftrightarrow \sup _{k}\left\|\varphi_{k} f\right\|_{\text {bmo }}<\infty
$$

and

$$
\|f\|_{\mathrm{bmo}} \approx \sup _{k}\left\|\varphi_{k} f\right\|_{\mathrm{bmo}}
$$

Proof. The Lipschitz bounds on $\varphi_{k}$ yield

$$
\left\|\varphi_{k} f\right\|_{\mathrm{bmo}} \leq C\|f\|_{\mathrm{bmo}}
$$

with $C$ independent of $k$, by Proposition 3.1. The converse inequality

$$
\|f\|_{\text {bmo }} \leq C \sup _{k}\left\|\varphi_{k} f\right\|_{\text {bmo }}
$$

follows directly from the identity

$$
f=\sum_{k} \varphi_{k} f=\sum_{\nu=1}^{K_{1}} \sum_{k \in \mathcal{S}_{\nu}} \varphi_{k} f,
$$

with $\mathcal{S}_{\nu}$ as in (2.19), the support conditions on $\left\{\varphi_{k} f\right\}$, and the definition of the bmo-norm.

From here we easily have the following counterparts to Proposition 2.3 and Corollary 2.4.

Proposition 3.3. We have, uniformly in $k \in \mathbb{Z}^{+}$,

$$
\left\|\varphi_{k} f\right\|_{\mathrm{bmo}(M)} \approx\left\|\left(\varphi_{k} f\right) \circ \operatorname{Exp}_{p_{k}}\right\|_{\mathrm{bmo}\left(\mathbb{R}^{n}\right)} .
$$

Proof. This follows via the equivalence between the use of (3.1) and (3.7), and the equivalence between the use of (3.3) and (3.11)-(3.12).

Corollary 3.4. In the setting of Proposition 3.2,

$$
\|f\|_{\mathrm{bmo}(M)} \approx \sup _{k}\left\|\left(\varphi_{k} f\right) \circ \operatorname{Exp}_{p_{k}}\right\|_{\mathrm{bmo}\left(\mathbb{R}^{n}\right)}
$$

4. $\mathfrak{h}^{1}-$ bmo duality

As before, we take $M$ to be a complete Riemannian manifold with bounded geometry, of dimension $n$. Here our aim is to prove the following extension of (1.12). 
Proposition 4.1. We have

$$
\mathfrak{h}^{1}(M)^{\prime}=\operatorname{bmo}(M) .
$$

Proof. To begin, we take $f \in \mathfrak{h}^{1}(M)$ and $g \in \operatorname{bmo}(M)$ and show the pairing $\langle f, g\rangle$ is well defined. Let $\left\{\varphi_{k}: k \in \mathbb{Z}^{+}\right\}$be a tame partition of unity, as in (1.25)-(1.27). Also take a bounded family $\psi_{k} \in C_{0}^{\infty}\left(B_{2}\left(p_{k}\right)\right) \approx C_{0}^{\infty}\left(B_{2}(0)\right)$ such that $\psi_{k} \equiv 1$ on $\operatorname{supp} \varphi_{k}$. We attempt to define $\langle f, g\rangle$ as

$$
\langle f, g\rangle=\sum_{k}\left\langle f_{k}, \psi_{k} g\right\rangle, \quad f_{k}=\varphi_{k} f
$$

By Proposition 2.2 we have

$$
\tilde{f}_{k}=f_{k} \circ \operatorname{Exp}_{p_{k}} \in \mathfrak{h}^{1}\left(\mathbb{R}^{n}\right), \quad \mathbb{R}^{n} \approx T_{p_{k}} M,
$$

and as in Proposition 3.2 we have

$$
\tilde{g}_{k}=\left(\psi_{k} g\right) \circ \operatorname{Exp}_{p_{k}} \in \operatorname{bmo}\left(\mathbb{R}^{n}\right) .
$$

The volume element on $B_{4}\left(p_{k}\right) \subset M$ pulls back to

$$
A_{k} \in C^{\infty}\left(B_{4}(0)\right) \text {, bounded uniformly in } k \text {. }
$$

Hence we can set

$$
\left\langle f_{k}, \psi_{k} g\right\rangle=\left\langle A_{k} \tilde{f}_{k}, \tilde{g}_{k}\right\rangle
$$

the pairing on the right side defined by the duality $\mathfrak{h}^{1}\left(\mathbb{R}^{n}\right)^{\prime}=\operatorname{bmo}\left(\mathbb{R}^{n}\right)$, proven in Corollary 1 of $[\mathrm{G}]$ (making essential use of the result $H^{1}\left(\mathbb{R}^{n}\right)^{\prime}=\operatorname{BMO}\left(\mathbb{R}^{n}\right)$ from $[\mathrm{FS}])$. We have

$$
\begin{aligned}
\left|\left\langle f_{k}, \psi_{k} g\right\rangle\right| & \leq\left\|A_{k} \tilde{f}_{k}\right\|_{\mathfrak{h}^{1}\left(\mathbb{R}^{n}\right)}\left\|\tilde{g}_{k}\right\|_{\mathrm{bmo}\left(\mathbb{R}^{n}\right)} \\
& \leq C\left\|f_{k}\right\|_{\mathfrak{h}^{1}(M)}\|g\|_{\mathrm{bmo}(M)},
\end{aligned}
$$

the last inequality by Proposition 2.1 (with $M=\mathbb{R}^{n}$, applied to $\tilde{f}_{k} \mapsto A_{k} \tilde{f}_{k}$ ), together with Propositions 2.3, 3.1, and 3.3. Then we apply Proposition 2.2 to see that the series (4.2) converges and

$$
|\langle f, g\rangle| \leq C\|f\|_{\mathfrak{h}^{1}}\|g\|_{\text {bmo }}
$$

This shows that $\operatorname{bmo}(M) \subset \mathfrak{h}^{1}(M)^{\prime}$. 
For the converse, we let $\omega$ be a continuous linear functional on $\mathfrak{h}^{1}(M)$, and take up the task of associating an element $g_{\omega} \in \mathrm{bmo}(M)$. To start, we apply Proposition 2.2 and for $f \in \mathfrak{h}^{1}(M)$ write

$$
\omega(f)=\sum_{k} \omega\left(\varphi_{k} f\right)=\sum_{k} \omega_{k}(f)
$$

where $\omega_{k}(f)=\omega\left(\varphi_{k} f\right)$. Another appeal to Corollary 1 of $[\mathrm{G}]$ and arguments similar to those done above give

$$
\omega_{k}(f)=\left\langle f, g_{k}\right\rangle
$$

with

$$
\operatorname{supp} g_{k} \subset B_{1}\left(p_{k}\right), \quad\left\|g_{k}\right\|_{\operatorname{bmo}(M)} \leq C\left\|\omega_{k}\right\|_{\mathfrak{h}^{1}(M)^{\prime}} \leq C^{\prime}\|\omega\|_{\mathfrak{h}^{1}(M)^{\prime}}
$$

The properties listed in (4.11) (in concert with (1.26)) in turn give

$$
g_{\omega}=\sum_{k} g_{k} \in \operatorname{bmo}(M), \quad\left\|g_{\omega}\right\|_{\mathrm{bmo}} \leq C\|\omega\|_{\mathfrak{h}^{1}(M)^{\prime}}
$$

satisfying

$$
\omega(f)=\left\langle f, g_{\omega}\right\rangle
$$

This completes the proof of Proposition 4.1.

REMARK. From Proposition 4.1 we deduce that the multipliers $f \mapsto a f$ on $\mathfrak{h}^{1}(M)$ given by Proposition 2.1 are also multipliers on $\operatorname{bmo}(M)$, and the multipliers on $\operatorname{bmo}(M)$ given by Proposition 3.1 are also multipliers on $\mathfrak{h}^{1}(M)$. This is of some interest, since the classes of multipliers treated in these two propositions are slightly different.

\section{Atomic theory (ionic theory) of $\mathfrak{h}^{1}(M)$}

In the theory of $H^{1}\left(\mathbb{R}^{n}\right)$, an atom is a function $a$ satisfying, for some $p \in \mathbb{R}^{n}, r \in$ $(0, \infty)$,

$$
\operatorname{supp} a \subset B_{r}(p), \quad\|a\|_{L^{\infty}} \leq r^{-n}
$$

and

$$
\int a d x=0 .
$$


It is not hard to show that for such $a$

$$
\|\mathcal{G} a\|_{L^{1}} \leq C, \quad \text { independent of } p, r,
$$

and hence

$$
f=\sum \lambda_{j} a_{j}, a_{j} \text { atoms } \Longrightarrow\|f\|_{H^{1}} \leq C \sum\left|\lambda_{j}\right|
$$

The converse result is that each $f \in H^{1}\left(\mathbb{R}^{n}\right)$ has such an atomic decomposition; cf. [St] for a treatment, due originally to R. Coifman for $n=1$ and $\mathrm{R}$. Latter for $n>1$. In $[\mathrm{G}]$ the atomic decomposition of $\mathfrak{h}^{1}\left(\mathbb{R}^{n}\right)$ was given in terms of atoms which, this time, satisfy (5.1)-(5.2) for $r \leq 1$ but only (5.1) for $r>1$.

Note that if $a$ is an atom satisfying (5.1)-(5.2) and $\varphi$ is Lipschitz, with $\|\varphi\|_{L^{\infty}} \leq 1$ and $\|\varphi\|_{\text {Lip }} \leq L$, then $b=\varphi a$ satisfies

$$
\operatorname{supp} b \subset B_{r}(p), \quad\|b\|_{L^{\infty}} \leq r^{-n},
$$

and

$$
\left|\int b d x\right| \leq V\left(B_{1}(p)\right) L(r \wedge 1)
$$

Adapting material from Appendix A of [MT], if $r \in(0,1]$, we call such $b$ an ion. More generally, if $b$ satisfies (5.5)-(5.6), we can write

$$
b=a+h,
$$

with

$$
h=b_{B_{r}(p)} \chi_{B_{r}(p)}, \quad\left|b_{B_{r}(p)}\right| \leq A_{n} L r^{1-n},
$$

with $a$ satisfying (5.1), up to a factor of $1+A_{n} L$. For such a function it is not hard to show that

$$
\left\|\mathcal{G}^{b} h\right\|_{L^{1}} \leq C L, \quad C \text { independent of } p, r \in(0,1]
$$

In concert with (5.3) this gives

$$
\left\|\mathcal{G}^{b} b\right\|_{L^{1}} \leq C(L+1)
$$

Hence, for ions satisfying (5.5)-(5.6) (with fixed $L<\infty$ and with $r \in(0,1]$ ), we have

$$
f=\sum \lambda_{j} b_{j}, b_{j} \text { ions } \Longrightarrow\|f\|_{\mathfrak{h}^{1}} \leq C(L+1) \sum\left|\lambda_{j}\right|
$$


The existence of an ionic decomposition of a general $f \in \mathfrak{h}^{1}\left(\mathbb{R}^{n}\right)$ follows from the atomic decomposition of $[\mathrm{G}]$ mentioned above.

We move now to the setting of a complete $n$-dimensional Riemannian manifold $M$ with bounded geometry (and with metric tensor satisfying (1.19)-(1.24)).

Definition. An ion (of ionic norm $\leq 2$ ) is a function $b$ on $M$ satisfying the following properties:

$$
\begin{aligned}
\operatorname{supp} b & \subset B_{r}(p) \text { for some } p \in M, r \in(0,1] \\
\|b\|_{L^{\infty}} & \leq r^{-n} \\
\left|\int b d V\right| & \leq r
\end{aligned}
$$

The results discussed above in concert with the results of $\S 2$ yield the following.

Proposition 5.1. If $\left\{b_{j}: j \in \mathbb{Z}^{+}\right\}$are ions, then

$$
\begin{aligned}
& f=\sum \lambda_{j} b_{j}, \sum\left|\lambda_{j}\right|<\infty \Longrightarrow \\
& f \in \mathfrak{h}^{1}(M) \text { and }\|f\|_{\mathfrak{h}^{1}} \leq C \sum\left|\lambda_{j}\right| .
\end{aligned}
$$

Conversely, if $f \in \mathfrak{h}^{1}(M)$, then there exist ions $b_{j}$ and $\lambda_{j} \in \mathbb{C}$ such that

$$
f=\sum \lambda_{j} b_{j}, \quad \sum\left|\lambda_{j}\right| \leq C\|f\|_{\mathfrak{h}^{1}}
$$

6. Action of $(\lambda I-\Delta)^{i t}$ and other pseudodifferential operators on bmo, $\mathfrak{h}^{1}$, and $L^{p}$

We take $M$ as in $\S \S 1-5$, particularly enforcing (1.19)-(1.24). Our first goal is to prove the following.

Proposition 6.1. With $M$ as above, let $\Delta$ be the Laplace-Beltrami operator on M. There exist $\lambda_{0}=\lambda_{0}(M) \in(0, \infty)$ such that whenever $\lambda \geq \lambda_{0}$,

$$
(\lambda I-\Delta)^{i t}: \operatorname{bmo}(M) \longrightarrow \operatorname{bmo}(M),
$$

with uniformly bounded operator norms for $|t| \leq 1$, hence, with $C=C(M, \lambda)$,

$$
\left\|(\lambda I-\Delta)^{i t} f\right\|_{\mathrm{bmo}} \leq C e^{C|t|}\|f\|_{\mathrm{bmo}}
$$

To begin the analysis, we write

$$
(\lambda I-\Delta)^{i t}=\Phi_{-i t, \lambda}(\sqrt{-\Delta}),
$$


where

$$
\Phi_{-i t, \lambda}(\zeta)=\left(\zeta^{2}+\lambda\right)^{i t}
$$

Results of $[\mathrm{CGT}]$ apply to analyze the integral kernel $K_{t, \lambda}(x, y)$ in

$$
(\lambda I-\Delta)^{i t} f(x)=\int_{M} K_{t, \lambda}(x, y) f(y) d V(y)
$$

They are described as follows. Given $W>0, m \in \mathbb{R}$, we say

$$
\Phi \in \mathcal{S}_{W}^{m}
$$

provided $\Phi$ is holomorphic and even on the strip

$$
\Omega_{W}=\{\zeta \in \mathbb{C}:|\operatorname{Im} \zeta|<W\}
$$

and satisfies $S_{1,0}^{m}$ estimates on $\Omega_{W}$ :

$$
\left|\Phi^{(j)}(\zeta)\right| \leq C_{j}(1+|\zeta|)^{m-j}, \quad \zeta \in \Omega_{W}
$$

Compare [CGT], Definition 3.1. As shown in $\S 3$ of [CGT] (cf. (3.45)), with an improvement given by (1.12) of [T3], if $K_{\Phi}$ is the integral kernel of $\Phi(\sqrt{-\Delta})$,

$$
\Phi \in \mathcal{S}_{W}^{m} \Longrightarrow\left|K_{\Phi}(x, y)\right| \leq C_{k} d(x, y)^{-k} e^{-W d(x, y)}, \quad \text { for } \quad d(x, y) \geq 1, k \in \mathbb{Z}^{+}
$$

when $M$ has bounded geometry. Here $C$ depends on $M$ and finitely many of the constants in (6.8). Now $\Phi_{-i t, \lambda}$, given by (6.4), satisfies, for each $\delta \in(0, \lambda)$,

$$
\Phi_{-i t, \lambda} \in \mathcal{S}_{\sqrt{\lambda-\delta}}^{0}
$$

uniformly in $t \in[-1,1]$, so we deduce that the kernel $K_{t, \lambda}$ in $(6.5)$ satisfies the estimate

$$
\left|K_{t, \lambda}(x, y)\right| \leq C e^{-\sqrt{\lambda-\delta} d(x, y)}, \quad \text { for } \quad d(x, y) \geq 1
$$

with $C=C(M, \lambda, \delta)$, independent of $t \in[-1,1]$.

The near diagonal behavior is covered by the following result. To state it, first for each $p \in M$, use $\operatorname{Exp}_{p}: T_{p} M \rightarrow M$, satisfying (1.19)-(1.24), to identify $B_{4}(p) \subset M$ with $B_{4}(0) \subset T_{p} M$, further identified with $B_{4}(0) \subset \mathbb{R}^{n}$, uniquely up to the action of $O(n)$. Thus functions supported on $B_{4}(p) \subset M$ are identified with functions supported on $B_{4}(0) \subset \mathbb{R}^{n}$. With this convention in place, we state the result, which, by (6.10), is a special case of Theorem 3.3 of [CGT]. 
Proposition 6.2. Given $p \in M$, take $\varphi_{j} \in C_{0}^{\infty}\left(B_{4}(0)\right) \approx C_{0}^{\infty}\left(B_{4}(p)\right)$, and set $M_{\varphi_{j}} f=\varphi_{j} f$. Then, for each $\lambda>0$,

$$
M_{\varphi_{1}}(\lambda I-\Delta)^{i t} M_{\varphi_{2}} \in O P S_{1,0}^{0}\left(\mathbb{R}^{n}\right)
$$

with uniform bounds for $t \in[-1,1], p \in M$.

Having these results, we now take $f \in \operatorname{bmo}(M)$ and estimate $g_{t}=(\lambda I-\Delta)^{i t} f$. It suffices to show that for each $p \in M$, with $B_{1}=B_{1}(p)$,

$$
\left\|g_{t}^{\#}\right\|_{L^{\infty}\left(B_{1}\right)} \leq C\|f\|_{\mathrm{bmo}}, \quad\left\|g_{t}\right\|_{L^{1}\left(B_{1}\right)} \leq C\|f\|_{\mathrm{bmo}}
$$

with $C$ independent of $p$ and of $t \in[-1,1]$. We also set $B_{r}=B_{r}(p)$. Take $\varphi \in C_{0}^{\infty}(M)$ such that

$$
\varphi=1 \text { on } B_{2}, \quad \operatorname{supp} \varphi \subset B_{3},
$$

and write

$$
f=\varphi f+(1-\varphi) f
$$

We first estimate $(\lambda I-\Delta)^{i t}(1-\varphi) f$ on $B_{1}$. To do this, we use (6.11) together with the following well known volume estimate. (See [CGT], Proposition 4.1, for a stronger result.)

Lemma 6.3. Given a Riemannian manifold $M$ with bounded geometry, there exists $C_{0}=C_{0}(M), \mu_{0}=\mu_{0}(M)$, and $K_{0}=K_{0}(M)$ such that for each $p \in M, r \in(0, \infty)$,

$$
\operatorname{Vol}_{r}(p) \leq C_{0}(1+r)^{\mu_{0}} e^{K_{0} r} .
$$

Making use of this, we have

$$
\begin{aligned}
x \in B_{1} \Longrightarrow & \left|\int K_{t, \lambda}(x, y)(1-\varphi(y)) f(y) d V(y)\right| \\
& \leq C \int_{M \backslash B_{1}} e^{-\sqrt{\lambda-\delta} d(p, y)} d V(y)\|f\|_{\mathrm{bmo}} \\
& \leq C \int_{1}^{\infty} r^{\mu_{0}} e^{\left(K_{0}-\sqrt{\lambda-\delta}\right) r} d r\|f\|_{\mathrm{bmo}} .
\end{aligned}
$$

The first inequality in (6.17) results from (6.11) plus the fact that $\int_{B}|f| d V \leq$ $C\|f\|_{\text {bmo }}$, uniformly for all unit balls $B \subset M$. The last line in (6.17) is $\leq C\|f\|_{\text {bmo }}$ provided $\lambda>K_{0}^{2}+\delta$. Thus, to make Proposition 6.1 work, we require

$$
\lambda_{0}>K_{0}^{2}
$$


In such a case, we have an $L^{\infty}$ estimate on $B_{1}$ for $(\lambda I-\Delta)^{i t}(1-\varphi) f$.

It remains to estimate $(\lambda I-\Delta)^{i t} \varphi f$ on $B_{1}$. Let us fix $\varphi_{2} \in C_{0}^{\infty}\left(B_{3}(0)\right) \approx$ $C_{0}^{\infty}\left(B_{3}(p)\right)$ such that $\varphi_{2}=1$ on $\operatorname{supp} \varphi$. Then we can apply Proposition 6.2 to get

$$
M_{\varphi}(\lambda I-\Delta)^{i t} M_{\varphi_{2}} \in O P S_{1,0}^{0}\left(\mathbb{R}^{n}\right),
$$

with uniform bounds for $p \in M, t \in[-1,1]$. Theorem 4 of $[\mathrm{G}]$ implies this family of operators is uniformly bounded on $\mathfrak{h}^{1}\left(\mathbb{R}^{n}\right)$, and by duality we get

$$
\left\|\varphi_{2}(\lambda I-\Delta)^{i t} \varphi f\right\|_{\text {bmo }} \leq C\left\|\varphi_{2} f\right\|_{\text {bmo }} \leq C^{\prime}\|f\|_{\text {bmo }},
$$

where we also use Proposition 3.3. This completes the proof of Proposition 6.1.

A similar argument also proves:

Proposition 6.4. In the setting of Proposition 6.1,

$$
(\lambda I-\Delta)^{i t}: \mathfrak{h}^{1}(M) \longrightarrow \mathfrak{h}^{1}(M) .
$$

Furthermore, we have

$$
(\lambda I-\Delta)^{i t}: L^{p}(M) \longrightarrow L^{p}(M), \quad 1<p<\infty .
$$

REMARK. In fact, (6.22) is known in a much more general context; cf. [St0].

To put the results just established in context, and for use in later sections, it is useful to identify some distinguished classes of "pseudodifferential operators" on $M$ and record some of their mapping properties. To make the following definition, we retain the identification made in the statement of Proposition 6.2 of functions in $C_{0}^{\infty}\left(B_{4}(0)\right)$, where $B_{4}(0) \subset \mathbb{R}^{n}$, and functions in $C_{0}^{\infty}\left(B_{4}(p)\right)$, where $B_{4}(p) \subset M$, via the exponential map. Let $\varphi_{j} \in C^{\infty}\left(B_{4}(0)\right) \approx C_{0}^{\infty}\left(B_{4}(p)\right)$ be as in that proposition, and assume $\varphi_{j}=1$ on $B_{2}(0)$. Given an operator $P: C_{0}^{\infty}(M) \rightarrow \mathcal{D}^{\prime}(M)$, we will say

$$
P \in \Psi_{\#}^{m}(M)
$$

provided the following conditions hold. First, we assume its Schwartz kernel $K_{P} \in$ $\mathcal{D}^{\prime}(M \times M)$ satisfies

$$
\begin{gathered}
\operatorname{supp} K_{P} \subset\{(x, y) \in M \times M: d(x, y) \leq 1\}, \\
\text { sing supp } K_{P} \subset \operatorname{diag}(M \times M)=\{(x, x): x \in M\} .
\end{gathered}
$$

Next, we assume that for each $p \in M$,

$$
M_{\varphi_{1}} P M_{\varphi_{2}} \in O P S_{1,0}^{m}\left(\mathbb{R}^{n}\right),
$$

with uniform bounds, independent of $p \in M$. These conditions define (6.23).

In case $m=0$, the condition (6.25), with uniform bounds, implies uniform operator bounds for $M_{\varphi_{1}} P M_{\varphi_{2}}$ on $\mathfrak{h}^{1}\left(\mathbb{R}^{n}\right)$ and $\operatorname{bmo}\left(\mathbb{R}^{n}\right)$ (by Theorem 4 of $[\mathrm{G}]$ and duality), and on $L^{p}\left(\mathbb{R}^{n}\right)$ for $1<p<\infty$ (by Calderon-Zygmund theory). From here we get as before the following. 
Proposition 6.5. We have

$$
\begin{aligned}
P \in \Psi_{\#}^{0}(M) \Rightarrow & P: \mathfrak{h}^{1}(M) \rightarrow \mathfrak{h}^{1}(M), \\
& P: \operatorname{bmo}(M) \rightarrow \operatorname{bmo}(M), \\
& P: L^{p}(M) \rightarrow L^{p}(M), \quad 1<p<\infty .
\end{aligned}
$$

To proceed, given $W \in(0, \infty)$, we say

$$
P \in \Psi_{W}^{m}(M)
$$

provided we can write

$$
P=P^{\#}+P^{b}
$$

where $P^{\#} \in \Psi_{\#}^{m}(M)$ and $P^{b}$ has the form

$$
P^{b} f(x)=\int_{M} K^{b}(x, y) f(y) d V(y)
$$

where $K^{b}(x, y) \in C^{\infty}(M \times M)$ satisfies

$$
\left|K^{b}(x, y)\right| \leq C_{k}(1+d(x, y))^{-k} e^{-W d(x, y)}, \quad \forall k \in \mathbb{Z}^{+},
$$

and also such estimates hold for all $x$ and $y$-derivatives of $K^{b}(x, y)$ (say in local exponential coordinate systems). When (6.29)-(6.30) hold, we say

$$
P^{b} \in \Psi_{W}^{-\infty}(M)
$$

The main result in $\S 3$ of [CGT] can be summarized as follows:

Proposition 6.6. For $W \in(0, \infty), m \in \mathbb{R}$,

$$
\Phi \in \mathcal{S}_{W}^{m} \Longrightarrow \Phi(\sqrt{-\Delta}) \in \Psi_{W}^{m}(M)
$$

We recall that the proof of this result in $[\mathrm{CGT}]$ began with the representation

$$
\begin{aligned}
& \Phi(\sqrt{-\Delta}) f \\
& =\int_{-\infty}^{\infty} \widehat{\Phi}(t) \cos t \sqrt{-\Delta} f d t \\
& =\int_{-\infty}^{\infty} \widehat{\Phi}(t) \psi(t) \cos t \sqrt{-\Delta} f d t+\int_{-\infty}^{\infty} \widehat{\Phi}(t)(1-\psi(t)) \cos t \sqrt{-\Delta} f d t \\
& =\Phi^{\#}(\sqrt{-\Delta}) f+\Phi^{b}(\sqrt{-\Delta}) f,
\end{aligned}
$$


where in the second identity we take $\psi \in C_{0}^{\infty}(\mathbb{R})$ such that $\psi(t)=1$ for $|t| \leq 1 / 4,0$ for $|t| \geq 1 / 2$. As shown in $[\mathrm{CGT}]$, we have $\Phi^{\#}(\sqrt{-\Delta}) \in \Psi_{\#}^{m}(M)$, defined as in $(6.23)-(6.25)$, and $\Phi^{b}(\sqrt{-\Delta}) \in \Psi_{W}^{-\infty}(M)$. The result $\Phi^{\#}(\sqrt{-\Delta}) \in \Psi_{\#}^{m}(M)$ is established via finite propagation speed and a parametrix construction for the solution operator $\cos t \sqrt{-\Delta}$ to the wave equation. (The slightly greater precision of having the factors $C_{k}(1+d(x, y))^{-k}$ arises as in (1.12) of [T3].) Related results are also discussed in [T1] and in Chapter 5 of [T2].

As for boundedness on function spaces, given the estimate (6.16), we claim that if $P^{b}$ satisfies (6.29)-(6.30), i.e., $P^{b} \in \Psi_{W}^{-\infty}(M)$, then

$$
\begin{aligned}
W \geq K_{0} \Longrightarrow & P^{b}: \mathfrak{h}^{1}(M) \rightarrow \mathfrak{h}^{1}(M), \\
& P^{b}: \operatorname{bmo}(M) \rightarrow \operatorname{bmo}(M), \\
& P^{b}: L^{p}(M) \rightarrow L^{p}(M), \quad 1 \leq p \leq \infty .
\end{aligned}
$$

In fact, one has the following. Let $\left\{\varphi_{k}: k \in \mathbb{Z}^{+}\right\}$be a tame partition of unity, as in (1.27). Given $p, q \in[1, \infty]$, say

$$
f \in L_{(q)}^{(p)}(M) \Longleftrightarrow\left(\left\|\varphi_{k} f\right\|_{L^{q}}\right) \in \ell^{p},
$$

Note that $L_{(p)}^{(p)}(M)=L^{p}(M)$ for $p \in[1, \infty]$, and

$$
\operatorname{bmo}(M) \subset L_{(1)}^{(\infty)}(M), \quad L_{(\infty)}^{(1)}(M) \subset \mathfrak{h}^{1}(M) .
$$

The following results are straightforward, and imply (6.33):

$$
\begin{aligned}
P^{b} \in \Psi_{W}^{-\infty}(M), W \geq K_{0} \Longrightarrow & P^{b}: L_{(1)}^{(p)}(M) \rightarrow L_{(\infty)}^{(p)}(M), \forall p \in[1, \infty] \\
\Longrightarrow & P^{b}: L^{1}(M) \rightarrow \mathfrak{h}^{1}(M) \text { and } \\
& P^{b}: \operatorname{bmo}(M) \rightarrow L^{\infty}(M) .
\end{aligned}
$$

Together with Proposition 6.5, (6.33) gives:

Proposition 6.7. Given $K_{0}$ as in (6.16),

$$
\begin{aligned}
W \geq K_{0}, P \in \Psi_{W}^{0}(M) \Rightarrow & P: \mathfrak{h}^{1}(M) \rightarrow \mathfrak{h}^{1}(M) \\
& P: \operatorname{bmo}(M) \rightarrow \operatorname{bmo}(M), \\
& P: L^{p}(M) \rightarrow L^{p}(M), \quad 1<p<\infty .
\end{aligned}
$$

In particular, these mapping properties hold for $\Phi(\sqrt{-\Delta})$, given $\Phi \in \mathcal{S}_{W}^{0}, W>K_{0}$.

REMARK. A sharper $L^{p}$-boundedness result on $\Phi(\sqrt{-\Delta})$ was demonstrated in [T3], namely, for $p \in(1, \infty)$,

$$
\Phi \in \mathcal{S}_{W}^{0}, W \geq\left|\frac{1}{p}-\frac{1}{2}\right| \cdot K_{0} \Longrightarrow \Phi(\sqrt{-\Delta}): L^{p}(M) \rightarrow L^{p}(M) .
$$


In fact, the following more general result is proven in [T3]. Suppose $A \geq 0$ and

$$
\operatorname{Spec}(-\Delta) \subset[A, \infty) \text { on } L^{2}(M)
$$

Then, for $p \in(1, \infty)$,

$(6.38 \mathrm{~B}) \Phi \in \mathcal{S}_{W}^{0}, W \geq\left|\frac{1}{p}-\frac{1}{2}\right| \cdot K_{0}, L=\Delta+A \Longrightarrow \Phi(\sqrt{-L}): L^{p}(M) \rightarrow L^{p}(M)$.

The condition (6.38A) holds with $A>0$ for hyperbolic space and other symmetric spaces of noncompact type. See Appendices B and C for more on this.

For later use, we establish the following result on composition.

Proposition 6.8. Given $W \geq K_{0}$, we have

$$
P_{j} \in \Psi_{W}^{m_{j}}(M) \Longrightarrow P_{1} P_{2} \in \Psi_{W-K_{0} / 2}^{m_{1}+m_{2}}(M) .
$$

Proof. Write $P_{j}=P_{j}^{\#}+P_{j}^{b}$ with $P_{j}^{\#} \in \Psi_{\#}^{m_{j}}(M)$ and $P_{j}^{b} \in \Psi_{W}^{-\infty}(M)$. Furthermore, arrange that the Schwartz kernels of $P_{j}^{\#}$ are supported in $\{(x, y) \in M \times M$ : $d(x, y) \leq 1 / 2\}$. We claim that

$$
\begin{aligned}
P_{1}^{\#} P_{2}^{\#} & \in \Psi_{\#}^{m_{1}+m_{2}}(M), \\
P_{1}^{\#} P_{2}^{b}, P_{1}^{b} P_{2}^{\#} & \in \Psi_{W}^{-\infty}(M), \\
P_{1}^{b} P_{2}^{b} & \in \Psi_{W-K_{0} / 2}^{-\infty}(M) .
\end{aligned}
$$

These results imply $(6.39)$.

Of these results, (6.40) follows from standard Euclidean space pseudodifferential operator calculus, especially the composition results

$$
O P S_{1,0}^{m_{1}}\left(\mathbb{R}^{n}\right) \times O P S_{1,0}^{m_{2}}\left(\mathbb{R}^{n}\right) \longrightarrow O P S_{1,0}^{m_{1}+m_{2}}\left(\mathbb{R}^{n}\right)
$$

The first result in (6.41) follows from the fact that

$$
K_{P_{1}^{\#} P_{2}^{b}}(\cdot, y)=P_{1}^{\#} K_{P_{2}^{b}}(\cdot, y),
$$

plus standard pseudodifferential operator estimates, and the second part from the first, by passing to the adjoint.

This leaves (6.42). Note that

$$
\left|K_{P_{1}^{b} P_{2}^{b}}(x, y)\right| \leq C_{k} \int_{M}\langle d(x, z)\rangle^{-k}\langle d(z, y)\rangle^{-k} e^{-W[d(x, z)+d(z, y)]} d V(z)
$$


Write

$$
\begin{aligned}
M & =A \cup B \cup C, \quad C=M \backslash(A \cup B), \\
A & =\left\{z \in M: d(x, z) \leq \frac{1}{2} d(x, y)\right\}, \\
B & =\left\{z \in M: d(y, z) \leq \frac{1}{2} d(x, y)\right\} .
\end{aligned}
$$

Since $d(x, z)+d(y, z) \geq d(x, y)$ and $\langle d(x, z)\rangle\langle d(z, y)\rangle \geq C\langle d(x, y)\rangle$, we have

$$
\begin{aligned}
& \int_{A}\langle d(x, z)\rangle^{-k}\langle d(z, y)\rangle^{-k} e^{-W[d(x, z)+d(z, y)]} d V(z) \\
& \leq C_{k}\langle d(x, y)\rangle^{-k} e^{-W d(x, y)} \operatorname{Vol}(A) \\
& \leq C_{k}\langle d(x, y)\rangle^{-k+\mu_{0}} e^{-\left(W-K_{0} / 2\right) d(x, y)},
\end{aligned}
$$

the latter estimate by (6.16). There is a similar estimate on $\int_{B}$. Finally, since $d(z, y) \geq d(x, y) / 2$ on $C$,

$$
\begin{aligned}
& \int_{C}\langle d(x, z)\rangle^{-k}\langle d(z, y)\rangle^{-k} e^{-W[d(x, z)+d(z, y)]} d V(z) \\
& \leq C_{k}\langle d(x, y)\rangle^{-k / 2} e^{-W d(x, y) / 2} \int_{M \backslash A}\langle d(x, z)\rangle^{-k / 2} e^{-W d(x, z)} d V(z) \\
& \leq C_{k}\langle d(x, y)\rangle^{-k / 2} e^{-W d(x, y) / 2} \int_{d(x, y) / 2}^{\infty}\langle r\rangle^{\mu_{0}-k / 2} e^{-W r} e^{K_{0} r} d r \\
& =C_{k}\langle d(x, y)\rangle^{-k / 2} e^{-\left(W-K_{0} / 2\right) d(x, y)},
\end{aligned}
$$

provided $W \geq K_{0}$ (taking $k$ large enough). Similar estimates hold for derivatives of $K_{P_{1}^{b} P_{2}^{b}}(x, y)$. This completes the proof.

The following variant of Proposition 6.8 will also prove useful.

Proposition 6.9. Given $W>0$, we have

$$
P_{1} \in \Psi_{W}^{m_{1}}(M), P_{2} \in \Psi_{W+K_{0}}^{m_{2}}(M) \Longrightarrow P_{1} P_{2}, P_{2} P_{1} \in \Psi_{W}^{m_{1}+m_{2}}(M) .
$$

Proof. With $P_{j}=P_{j}^{\#}+P_{j}^{b}$ as before, the results (6.40)-(6.41) are readily verified, together with their analogues with the subscripts 1 and 2 interchanged. In place of (6.42), this time we claim

$$
P_{1}^{b} P_{2}^{b}, P_{2}^{b} P_{1}^{b} \in \Psi_{W}^{-\infty}(M)
$$

We treat $P_{1}^{b} P_{2}^{b}$; a similar argument will handle the other product. In place of (6.45), we have

$$
\left|K_{P_{1}^{b} P_{2}^{b}}(x, y)\right| \leq C_{k} \int_{M}\langle d(x, z)\rangle^{-k}\langle d(z, y)\rangle^{-k} e^{-W[d(x, z)+d(z, y)]} e^{-K_{0} d(z, y)} d V(z) .
$$


Using again the observations below (6.46), we see that the right side of (6.51) is

$$
\begin{aligned}
& \leq C_{k}\langle d(x, y)\rangle^{-k / 2} e^{-W d(x, y)} \int_{M}\langle d(z, y)\rangle^{-k / 2} e^{-K_{0} d(z, y)} d V(z) \\
& \leq C_{k}\langle d(x, y)\rangle^{-k / 2} e^{-W d(x, y)},
\end{aligned}
$$

the latter estimate by (6.16). There are similar estimates on derivatives, giving $(6.50)$.

\section{Local $L^{p}$ estimates}

The purpose of this section is to obtain a local version of the $L^{p}$ estimates in Theorem 5 of [FS], which will allow us to obtain global $L^{p}$ estimates in $\S 8$.

Here, let

$$
Q_{1}=\left\{x \in \mathbb{R}^{n}: 0 \leq x_{j} \leq 1,1 \leq j \leq n\right\} \subset \mathbb{R}^{n}
$$

For this section, we set

$$
f^{\#}(x)=\sup _{Q \in \mathcal{Q}(x)} \frac{1}{V(Q)} \int_{Q}\left|f(x)-f_{Q}\right| d x,
$$

where $V$ is Lebesgue measure, as usual, $f_{Q}$ is the mean of $f$ over $f_{Q}$, and

$$
\mathcal{Q}(x)=\left\{Q \subset Q_{1}: Q \text { cube, } Q \ni x\right\} .
$$

Our goal is to prove the following.

Proposition 7.1. For $p \in(1, \infty)$, there exists $C_{n, p}<\infty$ with the following property. Given $f \in L^{1}\left(Q_{1}\right)$ such that

$$
\|f\|_{L^{1}\left(Q_{1}\right)} \leq 1, \quad\left\|f^{\#}\right\|_{L^{p}\left(Q_{1}\right)} \leq 1
$$

it follows that

$$
\|f\|_{L^{p}\left(Q_{1}\right)} \leq C_{n, p}
$$

To begin the proof, for $\alpha \in[1, \infty)$, subdivide $Q_{1}$ dyadically, into $2^{n}$ cubes of edge $1 / 2$, and denote by $Q_{j}^{\alpha}$ any such cubes for which

$$
\alpha<\frac{1}{V\left(Q_{j}^{\alpha}\right)} \int_{Q_{j}^{\alpha}}|f| d x \leq 2^{n} \alpha .
$$


For those dyadic cubes for which (7.6) fails, subdivide these dyadically, retaining those for which (7.6) holds, and continue this process, obtaining a family $\left\{Q_{j}^{\alpha}\right\}$ for which (7.6) holds. Note that

$$
|f(x)| \leq \alpha \text { a.e. on } Q_{1} \backslash \bigcup_{j} Q_{j}^{\alpha}
$$

Note that if $\beta>\alpha$ the cubes in $\left\{Q_{j}^{\beta}\right\}$ are sub-cubes of cubes in $\left\{Q_{j}^{\alpha}\right\}$. Set

$$
\mu(\alpha)=\sum_{j} V\left(Q_{j}^{\alpha}\right)
$$

Parallel to (4.4) of [FS], we aim to show that

$$
\mu(\alpha) \leq V\left(\left\{x \in Q_{1}: f^{\#}(x)>\frac{\alpha}{A}\right\}\right)+\frac{2}{A} \mu\left(2^{-n-1} \alpha\right)
$$

whenever

$$
\alpha \geq 2^{n+1}, \quad A \geq 1 \text {. }
$$

The proof is similar to that of [FS]. Fix a cube $Q_{0}=Q_{j_{0}}^{\alpha / 2^{n+1}}$ and look at all the cubes $Q_{j}^{\alpha} \subset Q_{0}$. Consider two cases:

CASE I: $Q_{0} \subset\left\{x: f^{\#}(x)>\alpha / A\right\}$. In this case,

$$
\sum_{Q_{j}^{\alpha} \subset Q_{0}} V\left(Q_{j}^{\alpha}\right) \leq V\left(Q_{0} \cap\left\{x: f^{\#}(x) \geq \frac{\alpha}{A}\right\}\right) .
$$

CASE II: $Q_{0} \not \subset\left\{x: f^{\#}(x)>\alpha / A\right\}$. In this case,

$$
\frac{1}{V\left(Q_{0}\right)} \int_{Q_{0}}\left|f(x)-f_{Q_{0}}\right| d x \leq \frac{\alpha}{A} .
$$

Now (7.6) implies both $\left|f_{Q_{0}}\right| \leq \alpha / 2$ and $|f|_{Q_{j}^{\alpha}}>\alpha$. Hence

$$
\int_{Q_{j}^{\alpha}}\left|f(x)-f_{Q_{0}}\right| d x \geq \frac{\alpha}{2} V\left(Q_{j}^{\alpha}\right),
$$

for each $Q_{j}^{\alpha} \subset Q_{0}$ of the form described above. Now sum (7.13) over all such cubes and compare the result to (7.12). This yields, in Case II,

$$
\sum_{Q_{j}^{\alpha} \subset Q_{0}} V\left(Q_{j}^{\alpha}\right) \leq \frac{2}{A} V\left(Q_{0}\right) .
$$


Now sum over all the cubes $Q_{0}$, i.e., over all the cubes in $\left\{Q_{j}^{\alpha / 2^{n+1}}\right\}$, taking into account the estimates (7.11) in Case I and (7.14) in Case II, to obtain the asserted estimate (7.9).

Next, parallel to (4.8) of [FS], we bring in

$$
\lambda(\alpha)=V\left(\left\{x \in Q_{1}: M f(x)>\alpha\right\}\right),
$$

where

$$
M f(x)=\sup _{Q \in \mathcal{Q}(x)} \frac{1}{V(Q)} \int_{Q}|f| d x .
$$

Clearly $M f(x)>\alpha$ whenever $x \in Q_{j}^{\alpha}$, so

$$
\mu(\alpha) \leq \lambda(\alpha), \quad \forall \alpha \in[1, \infty)
$$

We next aim to prove

$$
\lambda\left(\left(1+8^{n}\right) \alpha\right) \leq 2^{n} \mu(\alpha), \quad \forall \alpha \in[1, \infty) .
$$

To get this, we bring in the following notation. Given a cube $Q$, let $\widetilde{Q}$ and $\widehat{Q}$ denote the concentric cubes dilated by factors of 2 and 4 , respectively. Now, take $\left\{Q_{j}^{\alpha}\right\}$ as above, and consider $x \in Q_{1} \backslash \cup_{j} \widetilde{Q}_{j}^{\alpha}$. Let $Q \in \mathcal{Q}(x)$, defined by (7.3). Consider

$$
\int_{Q}|f(y)| d y=\int_{Q \cap\left(\cup_{j} Q_{j}^{\alpha}\right)}|f(y)| d y+\int_{Q \backslash\left(\cup_{j} Q_{j}^{\alpha}\right)}|f(y)| d y .
$$

Since $|f| \leq \alpha$ on $Q \backslash\left(\cup_{j} Q_{j}^{\alpha}\right)$, the last integral in (7.19) is $\leq \alpha V(Q)$. For the first integral on the right side of (7.19), we use the fact that

$$
\begin{aligned}
& \text { Given } x \in Q \backslash \widetilde{Q}_{j}^{\alpha}, \quad Q \cap Q_{j}^{\alpha} \neq \emptyset \Rightarrow Q \not \subset \widetilde{Q}_{j}^{\alpha} \\
& \Rightarrow Q_{j}^{\alpha} \subset \widehat{Q} \text {. }
\end{aligned}
$$

Hence

$$
\begin{aligned}
\int_{Q \cap\left(\cup_{j} Q_{j}^{\alpha}\right)}|f(y)| d y & \leq \sum_{Q_{j}^{\alpha} \subset \widehat{Q}} \int_{Q_{j}^{\alpha}}|f(y)| d y \\
& \leq \sum_{Q_{j}^{\alpha} \subset \widehat{Q}} 2^{n} \alpha V\left(Q_{j}^{\alpha}\right) \\
& \leq 8^{n} \alpha V(Q) .
\end{aligned}
$$


Thus, in (7.19), we have

$$
\int_{Q}|f(y)| d y \leq\left(1+8^{n}\right) \alpha V(Q)
$$

Since this is true for all $Q \in \mathcal{Q}(x)$, we deduce that

$$
x \in Q_{1} \backslash \bigcup_{j} \widetilde{Q}_{j}^{\alpha} \Rightarrow M f(x) \leq\left(1+8^{n}\right) \alpha, \quad \forall \alpha \geq 1
$$

Hence

$$
\left\{x \in Q_{1}: M f(x)>\left(1+8^{n}\right) \alpha\right\} \subset \bigcup_{j} \widetilde{Q}_{j}^{\alpha}, \quad \forall \alpha \geq 1
$$

and (7.18) is established.

Moving on towards the proof of (7.5), we will actually estimate $\|M f\|_{L^{p}\left(Q_{1}\right)}$. Note that

$$
\|M f\|_{L^{p}\left(Q_{1}\right)}^{p}=p \int_{0}^{\infty} \alpha^{p-1} \lambda(\alpha) d \alpha=\lim _{N \rightarrow \infty} p \int_{0}^{N} \alpha^{p-1} \lambda(\alpha) d \alpha .
$$

Furthermore, for $N \geq 16^{n+1}$,

$$
p \int_{0}^{N} \alpha^{p-1} \lambda(\alpha) d \alpha=p \int_{0}^{16^{n+1}} \alpha^{p-1} \lambda(\alpha) d \alpha+p \int_{16^{n+1}}^{N} \alpha^{p-1} \lambda(\alpha) d \alpha .
$$

To estimate the first integral on the right, we use

$$
\lambda(\alpha) \leq \frac{C}{\alpha}\|f\|_{L^{1}}
$$

which follows by weak $(1,1)$ boundedness of $f \mapsto M f$, to obtain

$$
\begin{aligned}
p \int_{0}^{16^{n+1}} \alpha^{p-1} \lambda(\alpha) d \alpha & \leq C p \int_{0}^{16^{n+1}} \alpha^{p-2} d \alpha\|f\|_{L^{1}} \\
& =\frac{C p}{p-1} 16^{(n+1)(p-1)}\|f\|_{L^{1}}
\end{aligned}
$$

To treat the second integral on the right side of (7.26), we bring in (7.18). We have

$$
\begin{aligned}
p \int_{16^{n+1}}^{N} \alpha^{p-1} \lambda(\alpha) d \alpha & =p 8^{(n+1) p} \int_{2^{n+1}}^{N / 8^{n+1}} \beta^{p-1} \lambda\left(8^{n+1} \beta\right) d \beta \\
& \leq p 2^{n} 8^{(n+1) p} \int_{2^{n+1}}^{N / 8^{n+1}} \beta^{p-1} \mu(\beta) d \beta
\end{aligned}
$$


Having this, we follow [FS] and study, for $M \geq 2^{n+1}$,

$$
I_{M}=p \int_{2^{n+1}}^{M} \alpha^{p-1} \mu(\alpha) d \alpha .
$$

Using (7.9), we have

$$
\begin{aligned}
I_{M} \leq & p \int_{2^{n+1}}^{M} \alpha^{p-1} V\left(\left\{x \in Q_{1}: f^{\#}(x)>\frac{\alpha}{A}\right\}\right) d \alpha \\
& +\frac{2}{A} p \int_{2^{n+1}}^{M} \alpha^{p-1} \mu\left(2^{-n-1} \alpha\right) d \alpha \\
\leq & A^{p}\left\|f^{\#}\right\|_{L^{p}\left(Q_{1}\right)}^{p}+\frac{2}{A} 2^{(n+1) p} p \int_{1}^{M / 2^{n+1}} \beta^{p-1} \mu(\beta) d \beta .
\end{aligned}
$$

Provided $M>4^{n+1}$, we write the last integral as

$$
\int_{1}^{2^{n+1}} \beta^{p-1} \mu(\beta) d \beta+\int_{2^{n+1}}^{M / 2^{n+1}} \beta^{p-1} \mu(\beta) d \beta .
$$

Since $\mu(\beta) \leq \lambda(\beta)$, we have as in (7.28) the estimate

$$
\int_{1}^{2^{n+1}} \beta^{p-1} \mu(\beta) d \beta \leq \frac{C}{p-1} 2^{(n+1)(p-1)}\|f\|_{L^{1}},
$$

while the last integral in (7.32), multiplied by $p$, is $\leq I_{M}$. Hence (7.31) yields

$$
I_{M} \leq A^{p}\left\|f^{\#}\right\|_{L^{p}}^{p}+\frac{2 C}{A} \frac{p}{p-1} 2^{(n+1)(2 p-1)}\|f\|_{L^{1}}+\frac{2 \cdot 2^{(n+1) p}}{A} I_{M} .
$$

This holds for each $A \geq 1$. Take $A=4 \cdot 2^{(n+1) p}$ to obtain

$$
\frac{1}{2} I_{M} \leq A^{p}\left\|f^{\#}\right\|_{L^{p}}^{p}+C_{n, p}\|f\|_{L^{1}}, \quad \forall M \geq 4^{n+1} .
$$

From here on, $C_{n, p}$ denotes various (unevaluated) constants. Bringing in (7.25)(7.30), we deduce

$$
\begin{aligned}
\|M f\|_{L^{p}}^{p} & \leq C_{n, p}\|f\|_{L^{1}}+\lim _{M \rightarrow \infty} C_{n, p} I_{M} \\
& \leq C_{n, p}\left(\|f\|_{L^{p}}^{p}+\|f\|_{L^{1}}\right),
\end{aligned}
$$

the last inequality by (7.35). This holds under the hypothesis (7.4). Hence the conclusion (7.5) is established, and Proposition 7.1 is proven.

The following is a straightforward corollary. 
Corollary 7.2. Given $p \in(1, \infty)$, if $f \in L^{1}\left(Q_{1}\right)$ and $f^{\#} \in L^{p}\left(Q_{1}\right)$, then $f \in$ $L^{p}\left(Q_{1}\right)$, and

$$
\|f\|_{L^{p}\left(Q_{1}\right)} \leq C_{n, p}\left(\left\|f^{\#}\right\|_{L^{p}\left(Q_{1}\right)}+\|f\|_{L^{1}\left(Q_{1}\right)}\right) .
$$

\section{Global $L^{p}$ estimates}

We return to the setting of a complete Riemannian manifold $M$ with bounded geometry, and as in (3.1)-(3.4) define the following operators:

$$
\begin{aligned}
& \mathcal{N} f(x)=f^{\#}(x)+\mathcal{N}_{0} f(x), \\
& f^{\#}(x)=\sup _{B \in \mathcal{B}(x)} \frac{1}{V(B)} \int_{B}\left|f-f_{B}\right| d V, \quad \mathcal{B}(x)=\left\{B_{r}(x): 0<r \leq 1\right\},
\end{aligned}
$$

$$
\mathcal{N}_{0} f(x)=\frac{1}{V\left(B_{1}(x)\right)} \int_{B_{1}(x)}|f| d V
$$

which in turn define the bmo-norm:

$$
\|f\|_{\text {bmo }}=\|\mathcal{N} f\|_{L^{\infty}}
$$

It is clear that

$$
\mathcal{N} f(x) \leq 3 \mathcal{M}_{1} f(x)=\sup _{0<r \leq 1} \frac{3}{V\left(B_{r}(x)\right)} \int_{B_{r}(x)}|f| d V
$$

and the Hardy-Littlewood estimates

$$
\left\|\mathcal{M}_{1} f\right\|_{L^{p}(M)} \leq A_{p}\|f\|_{L^{p}(M)}, \quad 1<p<\infty
$$

are readily established when $M$ has bounded geometry. Thus

$$
\|f\|_{L^{p}(M)} \leq C_{p}\|\mathcal{N} f\|_{L^{p}(M)}
$$

for $p \in(1, \infty)$.

Our next goal is to establish the converse: 
Proposition 8.1. Assume $1<p<\infty, f \in L_{\mathrm{loc}}^{1}(M)$, and $\mathcal{N} f \in L^{p}(M)$. Then $f \in L^{p}(M)$, and

$$
\|f\|_{L^{p}(M)} \leq B_{p}\|\mathcal{N} f\|_{L^{p}(M)}
$$

In connection with this, we recall the following results. First, Theorem 5 of [FS] gives

$$
\|f\|_{L^{p}(M)} \leq B_{p}\left\|f^{\#}\right\|_{L^{p}(M)}, \quad 1<p<\infty
$$

for $M=\mathbb{R}^{n}$, but when $f^{\#}$ is given, not by (8.2), but by (1.7)-(1.8). Second, Proposition 1 of $[\mathrm{I}]$ gives (8.9) for $M=\mathcal{H}^{n}$, hyperbolic space, and more generally when $M$ is a rank one symmetric space of noncompact type. It is noted in [I] that (8.9) fails for $M=\mathbb{R}^{n}$ when $f^{\#}$ is defined by (8.2). For a counterexample, [I] mentions the family of characteristic functions of large balls in $\mathbb{R}^{n}$. Of course, such a family of functions does not furnish a counterexample to (8.8) in case $M=\mathbb{R}^{n}$.

In order to interface with the results of $\S 7$, we next obtain some estimates on $\mathcal{N}_{0} f$. Note that $f \in L_{\text {loc }}^{1}(M) \Rightarrow \mathcal{N}_{0} f \in C(M)$.

Lemma 8.2. There exist $C=C(M)<\infty$ and $K=K(M)<\infty$ with the following properties. For each $x \in M$ there are points $y_{1}, \ldots, y_{K} \in B_{1}(x)$ such that

$$
\left\{B_{1 / 2}\left(y_{j}\right): 1 \leq j \leq K\right\} \text { covers } B_{1}(x)
$$

and for each $f \in L_{\mathrm{loc}}^{1}(M)$, there exist $y_{j}^{\prime} \in B_{1 / 2}\left(y_{j}\right)$ such that

$$
\mathcal{N}_{0} f\left(y_{j}^{\prime}\right) \leq C\left\|\mathcal{N}_{0} f\right\|_{L^{1}\left(B_{1}(x)\right)} .
$$

Proof. The existence of $K$ and $y_{j}$ satisfying (8.10) follows from the bounded geometry conditions on $M$. One can furthermore arrange that

$$
V\left(B_{1 / 2}\left(y_{j}\right) \cap B_{1}(x)\right) \geq c_{0},
$$

for some $c_{0}=c_{0}(M)>0$. Then Chebycheff's inequality guarantees the existence of $C<\infty$ (independent of $f$ ) and $y_{j}^{\prime} \in B_{1 / 2}\left(y_{j}\right)$ (depending on $f$ ) such that (8.11) holds.

The definition (8.3) implies

$$
\mathcal{N}_{0} f(x) \leq \sum_{j=1}^{K} \mathcal{N}_{0} f\left(y_{j}^{\prime}\right)
$$

so we have: 
Corollary 8.3. In the setting of Lemma 8.2,

$$
V\left(B_{1}(x)\right)^{-1}\|f\|_{L^{1}\left(B_{1}(x)\right)}=\mathcal{N}_{0} f(x) \leq C K\left\|\mathcal{N}_{0} f\right\|_{L^{1}\left(B_{1}(x)\right)} .
$$

For notational simplicity, let us replace $C K$ by $C$, and furthermore denote by $C$ various constants $C(M)$ in the estimates below.

Lemma 8.4. Let $\left\{B_{1}\left(q_{k}\right): k \in \mathbb{Z}^{+}\right\}$be a tame cover of $M$, i.e., assume (1.25)(1.26) hold. Then there exists $C=C(M)<\infty$ such that

$$
\sum_{k}\|f\|_{L^{1}\left(B_{1}\left(q_{k}\right)\right)}^{p} \leq C\left\|\mathcal{N}_{0} f\right\|_{L^{p}(M)}^{p}
$$

Proof. Starting with (8.14), we have

$$
\begin{aligned}
\sum_{k}\|f\|_{L^{1}\left(B_{1}\left(q_{k}\right)\right)}^{p} & \leq C_{1} \sum_{k}\left\|\mathcal{N}_{0} f\right\|_{L^{1}\left(B_{1}\left(q_{k}\right)\right)}^{p} \\
& \leq C_{2} \sum_{k}\left\|\mathcal{N}_{0} f\right\|_{L^{p}\left(B_{1}\left(q_{k}\right)\right)}^{p} \\
& \leq C\left\|\mathcal{N}_{0} f\right\|_{L^{p}(M)}^{p},
\end{aligned}
$$

the last inequality via (1.26).

In order to interface with $\S 7$, it also helps to recall from $(3.7)-(3.10)$ that we can replace $f^{\#}$ in (8.2) by $f^{s}$, defined by

$$
f^{s}(x)=\sup _{B \in \mathcal{B}(x)} \inf _{c_{B} \in \mathbb{C}} \frac{1}{V(B)} \int_{B}\left|f-c_{B}\right| d V,
$$

since

$$
f^{s}(x) \leq f^{\#}(x) \leq 2 f^{s}(x)
$$

Furthermore, as in (3.11)-(3.12), we can fix $a, b, c \in(0, \infty)$, with $a<b$, and replace $\mathcal{B}(x)$ by

$$
\widetilde{\mathcal{B}}(x)=\left\{Q_{r}^{\alpha}(x): 0<r \leq 1, \alpha \in \mathcal{A}\right\}
$$

where $Q_{r}^{\alpha}(x)$ is a family of measurable sets with the property that for each $r \in(0,1]$,

$$
V\left(Q_{r}^{\alpha}(x)\right) \geq c V\left(B_{r}(x)\right), \quad Q_{r}^{\alpha}(x) \subset B_{b r}(x), \quad \text { for all } \alpha,
$$

and

$$
B_{a r}(x) \subset Q_{r}^{\alpha}(x), \quad \text { for some } \alpha \text {. }
$$


We can make the replacement first in (8.17), and then, by another application of (8.18), we can also make this replacement in (8.2). Furthermore, if we denote

$$
f^{\sigma}(x)=\sup _{\widehat{\mathcal{B}}(x)} \frac{1}{V(B)} \int_{B}\left|f-f_{B}\right| d V
$$

where $\widehat{\mathcal{B}}(x)$ is a family of the form (8.15), satisfying (8.20), but not (8.21), we still have

$$
f^{\sigma}(x) \leq C f^{\#}(x)
$$

This allows us to bring in the result of $\S 7$ as follows. Assume $f \in L_{\text {loc }}^{1}(M), \mathcal{N} f \in$ $L^{p}(M)$. Given $x \in M$, let $Q$ be the cube in $T_{x} M$, of edge $1 / \sqrt{n}$, centered at $0 \in T_{x} M$, identified with a subset of $M$ via $\operatorname{Exp}_{x}: T_{x} M \rightarrow M$. Then the function $\left(\left.f\right|_{Q}\right)^{\#}$, with \# defined as in $\S 7$, has the form $\left.f^{\sigma}\right|_{Q}$, for a certain class $\widehat{\mathcal{B}}(x)$ for which (8.23) holds, with $f^{\#}$ given by (8.2). Hence (dilating the cubes as needed) Corollary 7.2 (plus (8.23)) yields

$$
\left\|\left.f\right|_{Q}\right\|_{L^{p}(Q)}^{p} \leq C\left(\left\|f^{\#}\right\|_{L^{p}(Q)}^{p}+\|f\|_{L^{1}(Q)}^{p}\right) .
$$

Summing over a family of such "cubes," tamely covering $M$, and taking (8.15) into account, we have the proof of (8.8).

\section{An interpolation result}

In this section we establish the following variant of Corollary 2 in [FS]. As usual, $M$ is a complete Riemannian manifold with bounded geometry.

Proposition 9.1. Given $p \in(1, \infty)$, assume

$$
T: L^{p}(M) \longrightarrow L^{p}(M), \quad\|T f\|_{L^{p}} \leq M_{1}\|f\|_{L^{p}}
$$

Assume also that

$$
T: L^{\infty}(M) \longrightarrow \operatorname{bmo}(M), \quad\|T f\|_{\mathrm{bmo}} \leq M_{0}\|f\|_{L^{\infty}} .
$$

Then, for $q \in(p, \infty)$, i.e., $q=p / \theta, \theta \in(0,1)$, we have

$$
T: L^{q}(M) \longrightarrow L^{q}(M), \quad\|T f\|_{L^{q}} \leq C M_{1}^{\theta} M_{0}^{1-\theta}\|f\|_{L^{q}} .
$$

Proof. Take $f \in L^{q}(M)$ and produce a holomorphic family $f_{z}$, for $z$ in

$$
\bar{\Omega}=\{z \in \mathbb{C}: 0 \leq \operatorname{Re} z \leq 1\}
$$


with values in $L^{p}(M)+L^{\infty}(M)$, such that

$$
f_{\theta}=f, \quad\left\|f_{z}\right\|_{L^{q(z)}} \leq C\|f\|_{L^{q}}, \quad q(z)=\frac{p}{\operatorname{Re} z}, q=\frac{p}{\theta} .
$$

Thus we have $L^{\infty}$ bounds on $f_{i t}$ and $L^{p}$ bounds on $f_{1+i t}, t \in \mathbb{R}$. For example, we can take $f_{z}=(f /|f|)|f|^{z / \theta}$. Now set

$$
F_{z}=e^{z^{2}} T f_{z}
$$

To proceed, it is convenient to mollify $F_{z}$ as follows. Pick $\varphi \in C_{0}^{\infty}(\mathbb{R}), \varphi(t)=1$ for $|t| \leq 1 / 2,0$ for $|t| \geq 1$, and set $\psi_{\varepsilon}(x)=\varphi\left(\varepsilon \operatorname{dist}^{2}\left(x, x_{0}\right)\right)$ for some fixed $x_{0} \in M$. Then set

$$
G_{z}(x)=G_{z}^{\varepsilon}(x)=\psi_{\varepsilon}(x) e^{\varepsilon \Delta} F_{z}(x)
$$

We drop the $\varepsilon$ and denote the family of functions on $M$ by $G_{z}$ for notational simplicity. We will obtain estimates for $G_{z}^{\varepsilon}$ that are independent of $\varepsilon$.

Now, taking a cue from [FS], we let $x \mapsto B(x)$ be a measurable assignment to each $x \in M$ of a ball $B(x) \in \mathcal{B}(x)$ (defined by (3.3)), we take

$$
\eta \in L^{\infty}(M \times M), \quad|\eta(x, y)| \equiv 1
$$

and we set

$$
G_{z}^{B, \eta}(x)=\frac{1}{V(B(x))} \int_{B(x)}\left[G_{z}(y)-\left(G_{z}\right)_{B(x)}\right] \eta(x, y) d V(y)
$$

Then

$$
G_{z}^{\#}(x)=\sup _{B, \eta}\left|G_{z}^{B, \eta}(x)\right|
$$

the sup over $B, \eta$ as described above, when $G_{z}^{\#}$ is defined as in (8.2). In addition, for $\eta$ as in (9.7), set

$$
N_{0}^{\eta} G_{z}(x)=\frac{1}{V\left(B_{1}(x)\right)} \int_{B_{1}(x)} G_{z}(y) \eta(x, y) d V(y) .
$$

We have

$$
\mathcal{N}_{0} G_{z}(x)=\sup _{\eta}\left|N_{0}^{\eta} G_{z}(x)\right|
$$

the sup being over $\eta$ as in (9.7), with $\mathcal{N}_{0}$ defined as in (8.3). 
Now we have the following estimates on $G_{z}^{B, \eta}$ :

$$
\begin{aligned}
& \left\|G_{i t}^{B, \eta}\right\|_{L^{\infty}} \leq\left\|G_{i t}^{\#}\right\|_{L^{\infty}} \leq\left\|G_{i t}\right\|_{\mathrm{bmo}} \leq C M_{0}\|f\|_{L^{q}} \\
& \left\|G_{1+i t}^{B, \eta}\right\|_{L^{p}} \leq\left\|G_{1+i t}^{\#}\right\|_{L^{p}} \leq C_{p}\left\|G_{1+i t}\right\|_{L^{p}} \leq C M_{1}\|f\|_{L^{q}} .
\end{aligned}
$$

The second inequality in (9.12) follows from the definition of bmo, and the second inequality in (9.13) holds because of (8.5)-(8.6). From here, the standard interpolation inequalities for the $L^{q}$-interpolation scale yield

$$
\left\|G_{\theta}^{B, \eta}\right\|_{L^{q}} \leq C M_{1}^{\theta} M_{0}^{1-\theta}\|f\|_{L^{q}}
$$

with $C$ independent of $x \mapsto B(x)$ and of $\eta$. Hence we have

$$
\left\|G_{\theta}^{\#}\right\|_{L^{q}} \leq C M_{1}^{\theta} M_{0}^{1-\theta}\|f\|_{L^{q}}
$$

Similarly we have the following estimates for $N_{0}^{\eta} G_{z}$ :

$$
\begin{gathered}
\left\|N_{0}^{\eta} G_{i t}\right\|_{L^{\infty}} \leq\left\|\mathcal{N}_{0} G_{i t}\right\|_{L^{\infty}} \leq\left\|G_{i t}\right\|_{\text {bmo }} \leq C M_{0}\|f\|_{L^{q}}, \\
\left\|N_{0}^{\eta} G_{1+i t}\right\|_{L^{p}} \leq\left\|\mathcal{N}_{0} G_{1+i t}\right\|_{L^{p}} \leq C\left\|G_{1+i t}\right\|_{L^{p}} \leq C M_{1}\|f\|_{L^{q}} .
\end{gathered}
$$

Again standard interpolation gives

$$
\left\|N_{0}^{\eta} G_{\theta}\right\|_{L^{q}} \leq C M_{1}^{\theta} M_{0}^{1-\theta}\|f\|_{L^{q}},
$$

with $C$ independent of the choice of $\eta$, hence

$$
\left\|\mathcal{N}_{0} G_{\theta}\right\|_{L^{q}} \leq C M_{1}^{\theta} M_{0}^{1-\theta}\|f\|_{L^{q}} .
$$

We are almost done with the proof of Proposition 9.1. Combining (9.15) and (9.19) yields

$$
\left\|\mathcal{N} G_{\theta}\right\|_{L^{q}} \leq C M_{1}^{\theta} M_{0}^{1-\theta}\|f\|_{L^{q}}
$$

and then Proposition 8.1 gives an estimate on $\left\|G_{\theta}\right\|_{L^{q}}$, yielding

$$
\left\|\psi_{\varepsilon} e^{\varepsilon \Delta} T f\right\|_{L^{q}} \leq C M_{1}^{\theta} M_{0}^{1-\theta}\|f\|_{L^{q}}
$$

with $C$ independent of $\varepsilon \in(0,1]$. Taking $\varepsilon \searrow 0$ then proves (9.3).

10. $L^{p}, \mathfrak{h}^{1}$, and bmo-Sobolev spaces 
As usual, $M$ is a Riemannian manifold with bounded geometry, satisfying (1.19)(1.24). We want to define and study the spaces $H^{s, p}(M), \mathfrak{h}^{s, 1}(M)$, and $\mathfrak{h}^{s, \infty}(M)$ of functions (or distributions) with $s$ derivatives in $L^{p}(M), \mathfrak{h}^{1}(M)$, and $\operatorname{bmo}(M)$, respectively. Related results can be found in Chapter 7 of [Tri].

Here is one natural definition of these spaces when $s=k$ is a positive integer. Let $\mathcal{V}^{1}(M)$ denote the space of smooth vector fields $X$ on $M$ with the property that, in each exponential coordinate system $\operatorname{Exp}_{q}: T_{q} M \supset B_{1}(0) \rightarrow B_{1}(q)$, there is a uniform bound (independent of $q$ ) on the coefficients of $X$ and, for each $k$, a uniform bound on all the derivatives of these coefficients of order $\leq k$. Let $\mathcal{V}^{k}(M)$ denote the set of linear combinations of operators of the form $L=X_{1} \cdots X_{j}$, with $X_{\nu} \in \mathcal{V}^{1}(M)$ and $j \leq k$. Then we can define

$$
\begin{aligned}
H^{k, p}(M) & =\left\{u \in L^{p}(M): L u \in L^{p}(M), \forall L \in \mathcal{V}^{k}(M)\right\}, \\
\mathfrak{h}^{k, 1}(M) & =\left\{u \in \mathfrak{h}^{1}(M): L u \in \mathfrak{h}^{1}(M), \forall L \in \mathcal{V}^{k}(M)\right\}, \\
\mathfrak{h}^{k, \infty}(M) & =\left\{u \in \operatorname{bmo}(M): L u \in \operatorname{bmo}(M), \forall L \in \mathcal{V}^{k}(M)\right\} .
\end{aligned}
$$

There are alternative characterizations of these spaces. For one, let $\left\{B_{1}\left(p_{\ell}\right): \ell \in\right.$ $\left.\mathbb{Z}^{+}\right\}$be a tame cover of $M$ and $\left\{\varphi_{\ell}: \ell \in \mathbb{Z}^{+}\right\}$a tame partition of unity, as defined in (1.25)-(1.27). Given a function $u$ on $M$, set

$$
u_{\ell}=\left(\varphi_{\ell} u\right) \circ \operatorname{Exp}_{p_{\ell}}
$$

a function supported on $B_{1}(0) \subset T_{p_{\ell}} M$, which we can identify with $B_{1}(0) \subset \mathbb{R}^{n}$, uniquely up to the action of an element of $O(n)$. Then (given $p<\infty$ )

$$
\begin{aligned}
& u \in H^{k, p}(M) \Leftrightarrow \sum_{\ell} \sum_{|\alpha| \leq k}\left\|D^{\alpha} u_{\ell}\right\|_{L^{p}\left(B_{1}(0)\right)}^{p}<\infty, \\
& u \in \mathfrak{h}^{k, 1}(M) \Leftrightarrow \sum_{\ell} \sum_{|\alpha| \leq k}\left\|D^{\alpha} u_{\ell}\right\|_{\mathfrak{h}^{1}(M)}<\infty, \\
& u \in \mathfrak{h}^{k, \infty}(M) \Leftrightarrow \sup _{\ell} \sum_{|\alpha| \leq k}\left\|D^{\alpha} u_{\ell}\right\|_{\operatorname{bmo}(M)}<\infty
\end{aligned}
$$

Of the results just stated, given the definitions (10.1)-(10.3), the result (10.5) is straightforward, and (10.6)-(10.7) follow readily from the results of $\S \S 2-3$, particularly Corollary 2.4 and Corollary 3.4.

We next define these Sobolev spaces for arbitrary index of regularity $s \in \mathbb{R}$, as

$$
\begin{aligned}
H^{s, p}(M) & =(\lambda I-\Delta)^{-s / 2} L^{p}(M), \\
\mathfrak{h}^{s, 1}(M) & =(\lambda I-\Delta)^{-s / 2} \mathfrak{h}^{1}(M), \\
\mathfrak{h}^{s, \infty}(M) & =(\lambda I-\Delta)^{-s / 2} \operatorname{bmo}(M),
\end{aligned}
$$


where we take $\lambda$ as in Proposition 6.1, i.e., a sufficiently large positive number. More precisely, as in (6.18), take $\lambda>K_{0}^{2}$, where $K_{0}$ is as in (6.16). From here on, we work under the condition

$$
1<p<\infty
$$

Of course, we need to show that when $s=k$ is a positive integer, (10.1)-(10.3) are equivalent to (10.8)-(10.10). Before tackling this, we first need to show that the right sides of (10.8)-(10.10) are well defined. This will follow from results obtained in $\S 6$. To begin, we write

$$
(\lambda I-\Delta)^{-s / 2}=\Phi_{s, \lambda}(\sqrt{-\Delta})
$$

where

$$
\Phi_{s, \lambda}(\zeta)=\left(\zeta^{2}+\lambda\right)^{-s / 2}
$$

With $\mathcal{S}_{W}^{m}$ defined as in (6.6)-(6.8), we have

$$
\Phi_{s, \lambda} \in \mathcal{S}_{W}^{-s}, \quad \forall W<\sqrt{\lambda} .
$$

Hence, by Proposition 6.6, given $\lambda>0$,

$$
(\lambda I-\Delta)^{-s / 2} \in \Psi_{W}^{-s}(M), \quad \forall W<\sqrt{\lambda} .
$$

We can now establish the following.

Proposition 10.1. Given $\lambda>K_{0}^{2}$,

$$
\begin{aligned}
(\lambda I-\Delta)^{-k / 2}: L^{p}(M) & \longrightarrow H^{k, p}(M), \\
(\lambda I-\Delta)^{-k / 2}: \mathfrak{h}^{1}(M) & \longrightarrow \mathfrak{h}^{k, 1}(M), \\
(\lambda I-\Delta)^{-k / 2}: \operatorname{bmo}(M) & \longrightarrow \mathfrak{h}^{k, \infty}(M),
\end{aligned}
$$

where the spaces on the right are defined by (10.1)-(10.3).

Proof. Note that $\mathcal{V}^{k}(M) \subset \Psi_{\#}^{k}(M)$. Hence, by (6.40)-(6.41),

$$
L \in \mathcal{V}^{k}(M) \Longrightarrow L(\lambda I-\Delta)^{-k / 2} \in \Psi_{W}^{0}(M), \quad \forall W<\sqrt{\lambda}
$$

As long as we can take $W \geq K_{0}$, we can apply Proposition 6.1 to conclude that such $L(\lambda I-\Delta)^{-k / 2}$ is bounded on $L^{p}(M), p \in(1, \infty)$, on $\mathfrak{h}^{1}(M)$, and on $\operatorname{bmo}(M)$, establishing (10.16)-(10.18).

At this point, we have the spaces defined on the right sides of (10.8)-(10.10) contained in the spaces defined in (10.1)-(10.3), when $s=k$ is a positive integer. 
To proceed, it will be convenient to know that

$$
(\lambda I-\Delta)^{-r / 2}(\lambda I-\Delta)^{-s / 2} f=(\lambda I-\Delta)^{-(r+s) / 2} f, \quad \forall r, s \in \mathbb{R},
$$

whenever $f \in L^{p}(M), 1<p<\infty$, or $f \in \mathfrak{h}^{1}(M)$, or $f \in \operatorname{bmo}(M)$. The result (10.20) for $f \in L^{2}(M)$ is a well known consequence of Hilbert space spectral theory. In that case, the self-duality of $L^{2}(M)$ extends to produce the duality

$$
\left((\lambda I-\Delta)^{-s / 2} L^{2}(M)\right)^{\prime}=(\lambda I-\Delta)^{s / 2} L^{2}(M), \quad \forall s \in \mathbb{R}
$$

Now, given that $(\lambda I-\Delta)^{-k / 2} L^{2}(M)$ is contained in $H^{k, 2}(M)$ as defined by (10.1), or by (10.5), we have

$$
(\lambda I-\Delta)^{-k / 2} L^{2}(M) \subset L^{\infty}(M), \quad \forall k>\frac{n}{2},
$$

and hence, by duality,

$$
L^{1}(M) \subset(\lambda I-\Delta)^{k / 2} L^{2}(M), \quad \forall k>\frac{n}{2},
$$

from which it follows that whenever $k>n / 2$,

$$
\begin{aligned}
& L^{p}(M) \subset(\lambda I-\Delta)^{k / 2} L^{2}(M), \quad \forall p \in(1,2], \\
& \mathfrak{h}^{1}(M) \subset(\lambda I-\Delta)^{k / 2} L^{2}(M) .
\end{aligned}
$$

We can now prove:

Lemma 10.2. The identity (10.20) holds for all $f \in L^{p}(M), 1<p<\infty$, for all $f \in \mathfrak{h}^{1}(M)$, and for all $f \in \operatorname{bmo}(M)$.

Proof. We have seen that (10.20) holds for all $f \in L^{2}(M)$. The result (10.24) implies (10.20) holds on $\mathfrak{h}^{1}(M)$ and on $L^{p}(M)$ for $p \in(1,2]$. The facts that $(10.20)$ holds on $\operatorname{bmo}(M)$ and on $L^{p}(M)$ for $p \in(2, \infty)$ follow by duality.

We are now prepared to prove:

Proposition 10.3. If $s=k$ is a positive integer, the spaces defined by (10.1)(10.3) coincide with those defined by (10.8)-(10.10) (assuming $p \in(1, \infty)$ ).

Proof. We have one set of inclusions. For the converse, assume $u$ has the property

$$
u, X_{1} \cdots X_{j} u \in \mathfrak{X}, \quad \forall j \leq k, X_{\nu} \in \mathcal{V}^{1}(M),
$$

where either $\mathfrak{X}=L^{p}(M), 1<p<\infty$, or $\mathfrak{X}=\mathfrak{h}^{1}(M)$, or $\mathfrak{X}=\operatorname{bmo}(M)$. We claim

$$
f=(\lambda I-\Delta)^{k / 2} u \in \mathfrak{X}
$$


If so, then, by (10.20),

$$
u=(\lambda I-\Delta)^{-k / 2} f
$$

and we are done.

The result (10.26) is elementary if $k=2 j$ is an even integer. Then $(\lambda I-\Delta)^{j}$ is a differential operator, and it is a finite linear combination of operators of the form appearing in (10.25). Now suppose $k=2 j+1$. The same argument shows that

$$
v=(\lambda I-\Delta)^{j} u
$$

has the property

$$
v, X v \in \mathfrak{X}, \quad \forall X \in \mathcal{V}^{1}(M) .
$$

If we can show that for such $v$,

$$
(\lambda I-\Delta)^{1 / 2} v \in \mathfrak{X},
$$

we will be done. To get this, write

$$
(\lambda I-\Delta)^{1 / 2} v=\Phi_{-1, \lambda}^{\#}(\sqrt{-\Delta}) v+\Phi_{-1, \lambda}^{b}(\sqrt{-\Delta}) v,
$$

with $\Phi_{-1, \lambda}^{\#}(\sqrt{-\Delta}) \in \Psi_{\#}^{1}(M)$ and $\Phi_{-1, \lambda}^{b}(\sqrt{-\Delta}) \in \Psi_{W}^{-\infty}(M)$, for all $W<\sqrt{\lambda}$. Estimates in (6.33) give

$$
\Phi_{-1, \lambda}^{b}(\sqrt{-\Delta}): \mathfrak{X} \longrightarrow \mathfrak{X},
$$

for such spaces $\mathfrak{X}$. It remains to show that

$$
\Phi_{-1, \lambda}^{\#}(\sqrt{-\Delta}) v \in \mathfrak{X},
$$

whenever $(10.29)$ holds. Indeed, since $P=\Phi_{-1, \lambda}^{\#}(\sqrt{-\Delta}) \in \Psi_{\#}^{1}(M)$, as defined in (6.23), pseudodifferential operator calculus allows us to write

$$
P=Q_{0}+\sum_{j=1}^{N} Q_{j} X_{j}
$$

with

$$
X_{1}, \ldots, X_{N} \in \mathcal{V}^{1}(M), \quad Q_{j} \in \Psi_{\#}^{0}(M) .
$$

Hence, if $v$ satisfies (10.29),

$$
P v=Q_{0} v+\sum_{j=1}^{N} Q_{j}\left(X_{j} v\right) \in \mathfrak{X},
$$

for $\mathfrak{X}=\mathfrak{h}^{1}(M), \operatorname{bmo}(M)$, or $L^{p}(M), 1<p<\infty$, by Proposition 6.5.

Having identified the spaces (10.1)-(10.3) with their counterparts in (10.8)(10.10) when $s=k \in \mathbb{N}$, we next show that for general $s \in \mathbb{R}$, the spaces (10.8)(10.10) are independent of the choice of $\lambda$, as long as $\lambda>K_{0}^{2}$. 
Proposition 10.4. Let $\mathfrak{X}=L^{p}(M), p \in(1, \infty)$, or $\mathfrak{X}=\mathfrak{h}^{1}(M)$, or $\mathfrak{X}=\operatorname{bmo}(M)$. Then, for each $s \in \mathbb{R}$,

$$
\mu, \lambda>K_{0}^{2} \Longrightarrow(\lambda I-\Delta)^{-s / 2} \mathfrak{X}=(\mu I-\Delta)^{-s / 2} \mathfrak{X} .
$$

Proof. Note that

$$
(\mu I-\Delta)^{s / 2}(\lambda I-\Delta)^{-s / 2}=\psi_{s, \mu, \lambda}(\sqrt{-\Delta}), \quad \psi_{s, \mu \lambda}(\zeta)=\left(\frac{\mu+\zeta^{2}}{\lambda+\zeta^{2}}\right)^{s / 2}
$$

and $\psi_{s, \mu, \lambda} \in \mathcal{S}_{W}^{0}$ for all $W<\min (\mu, \lambda)$. Hence

$$
(\mu I-\Delta)^{s / 2}(\lambda I-\Delta)^{-s / 2}: \mathfrak{X} \longrightarrow \mathfrak{X},
$$

by Propositions 6.6-6.7, with inverse $\psi_{s, \lambda, \mu}(\sqrt{-\Delta})$, so (10.39) is an isomorphism for each such $\mathfrak{X}$. This gives (10.37).

We next record how elements of $\Psi_{W}^{m}(M)$ act on these Sobolev spaces.

Proposition 10.5. Take $m, s \in \mathbb{R}$ and assume $W \geq K_{0}$. Then

$$
\begin{aligned}
P \in \Psi_{W}^{m}(M) \Rightarrow P: H^{s, p}(M) & \rightarrow H^{s-m, p}(M), \quad \forall p \in(1, \infty), \\
P: \mathfrak{h}^{s, 1}(M) & \rightarrow \mathfrak{h}^{s-m, 1}(M), \\
P: \mathfrak{h}^{s, \infty}(M) & \rightarrow \mathfrak{h}^{s-m, \infty}(M) .
\end{aligned}
$$

Proof. The results in (10.40) are equivalent to the existence of $\lambda>K_{0}^{2}$ such that

$$
Q=(\lambda I-\Delta)^{(s-m) / 2} P(\lambda I-\Delta)^{-s / 2}
$$

has the mapping properties

$$
\begin{aligned}
Q: L^{p}(M) & \rightarrow L^{p}(M), \quad p \in(1, \infty), \\
Q: \mathfrak{h}^{1}(M) & \rightarrow \mathfrak{h}^{1}(M), \\
Q: \operatorname{bmo}(M) & \rightarrow \operatorname{bmo}(M) .
\end{aligned}
$$

To get this, take $\lambda>\left(W+K_{0}\right)^{2}$, so $(\lambda I-\Delta)^{\sigma / 2} \in \Psi_{W+K_{0}}^{\sigma}(M)$. An application of Proposition 6.9 gives $P(\lambda I-\Delta)^{-s / 2} \in \Psi_{W}^{m-s}(M)$, and a second application gives $Q \in \Psi_{W}^{0}(M)$. Then the mapping properties in (10.42) follow from Proposition 6.7.

\section{Interpolations of type $\left(p, p^{\prime}\right)$}

Our goal here is to establish the following interpolation result, of potential use for dispersive estimates in PDE. 
Proposition 11.1. Take $s \in \mathbb{R}$. Assume we have a bounded operator

$$
R: L^{2}(M) \longrightarrow L^{2}(M), \quad R: L^{1}(M) \longrightarrow \mathfrak{h}^{s, \infty}(M),
$$

satisfying

$$
\|R f\|_{L^{2}} \leq M_{1}\|f\|_{L^{2}}, \quad\|R f\|_{\mathfrak{h}^{s, \infty}} \leq M_{0}\|f\|_{L^{1}}
$$

Then, for $\theta \in(0,1)$,

$$
R: L^{p(\theta)}(M) \longrightarrow H^{(1-\theta) s, p(\theta)^{\prime}}(M), \quad p(\theta)=\frac{2}{2-\theta}, p(\theta)^{\prime}=\frac{2}{\theta},
$$

and (with $C_{\theta} \in(0, \infty)$ independent of $R$ and of $f$ ),

$$
\|R f\|_{H^{(1-\theta) s, p(\theta)^{\prime}}} \leq C_{\theta} M_{1}^{\theta} M_{0}^{1-\theta}\|f\|_{L^{p(\theta)}} .
$$

The proof combines methods of $\S 9$ and Sobolev results of $\S 10$. To start, set

$$
\Omega=\{z \in \mathbb{C}: 0<\operatorname{Re} z<1\} .
$$

Given $\theta \in(0,1), f \in L^{2 /(2-\theta)}(M)$, take $f_{z}(x)$ holomorphic in $z \in \Omega$, bounded and continuous on $\bar{\Omega}$ with vaules in $L^{1}(M)+L^{2}(M)$, satisfying

$$
f_{\theta}=f, \quad\left\|f_{z}\right\|_{L^{p(z)}} \leq C\|f\|_{L^{p(\theta)}}, \quad p(z)=\frac{2}{2-\operatorname{Re} z} .
$$

Note that $f_{i t} \in L^{1}(M)$ and $f_{1+i t} \in L^{2}(M)$, for $t \in \mathbb{R}$. Now take $\lambda>K_{0}^{2}$ and set

$$
F_{z}(x)=e^{z^{2}}(\lambda I-\Delta)^{s(1-z) / 2} R f_{z}(x) .
$$

The hypotheses (11.1)-(11.2), plus results of $\S 6$ and $\S 10$, give

$$
F_{i t} \in \operatorname{bmo}(M), \quad F_{1+i t} \in L^{2}(M),
$$

and

$$
\left\|F_{i t}\right\|_{\mathrm{bmo}} \leq C M_{0}\|f\|_{L^{p(\theta)}}, \quad\left\|F_{1+i t}\right\|_{L^{2}} \leq C M_{1}\|f\|_{L^{p(\theta)}} .
$$

To proceed, it is convenient to mollify $F_{z}$ as follows (parallel to the construction leading to (9.6)). Pick $\psi_{\varepsilon}(x)=\varphi\left(\varepsilon d\left(x, x_{0}\right)^{2}\right)$ as in (9.6), and set

$$
G_{z}(x)=G_{z}^{\varepsilon}(x)=\psi_{\varepsilon}(x) e^{\varepsilon \Delta} F_{z}(x),
$$


with $F_{z}(x)$ as in (11.7). We often drop the $\varepsilon$ and denote the family of functions on $M$ by $G_{z}$ for notational simplicity; we will obtain estimates for $G_{z}^{\varepsilon}$ that will be independent of $\varepsilon$.

With $G_{z}$ defined as above, take $\eta$ as in (9.7), let $x \mapsto B(x)$ be a measurable assignment, with $B(x) \in \mathcal{B}(x)$, and define $G_{z}^{B, \eta}$ as in (9.8). As in (9.9), we have

$$
G_{z}^{\#}(x)=\sup _{B, \eta}\left|G_{z}^{B, \eta}(x)\right|
$$

Next define $N_{0}^{\eta} G_{z}$ as in (9.10), so, as in (9.11),

$$
\mathcal{N}_{0} G_{z}(x)=\sup _{\eta}\left|N_{0}^{\eta} G_{z}(x)\right|
$$

In the current setting, we have the following variants of the estimates (9.12)-(9.13),

$$
\begin{aligned}
\left\|G_{i t}^{B, \eta}\right\|_{L^{\infty}} & \leq\left\|G_{i t}^{\#}\right\|_{L^{\infty}} \leq\left\|G_{i t}\right\|_{\text {bmo }} \leq C M_{0}\|f\|_{L^{p(\theta)}} \\
\left\|G_{1+i t}^{B, \eta}\right\|_{L^{2}} & \leq\left\|G_{1+i t}^{\#}\right\|_{L^{2}} \leq C\left\|G_{1+i t}\right\|_{L^{2}} \leq C M_{1}\|f\|_{L^{p(\theta)}},
\end{aligned}
$$

for the same reasons used to justify $(9.12)-(9.13)$. Then standard interpolation gives

$$
\left\|G_{\theta}^{B, \eta}\right\|_{L^{p(\theta)^{\prime}}} \leq C M_{1}^{\theta} M_{0}^{1-\theta}\|f\|_{L^{p(\theta)}}
$$

parallel to (9.14), with $C$ independent of $x \mapsto B(x)$ and of $\eta$. Hence

$$
\left\|G_{\theta}^{\#}\right\|_{L^{p(\theta)^{\prime}}} \leq C M_{1}^{\theta} M_{0}^{1-\theta}\|f\|_{L^{p(\theta)}} .
$$

We also have the following variants of (9.16)-(9.17):

$$
\begin{aligned}
\left\|N_{0}^{\eta} G_{i t}\right\|_{L^{\infty}} & \leq\left\|\mathcal{N}_{0} G_{i t}\right\|_{L^{\infty}} \leq\left\|G_{i t}\right\|_{\text {bmo }} \leq C M_{0}\|f\|_{L^{p(\theta)}} \\
\left\|N_{0}^{\eta} G_{1+i t}\right\|_{L^{2}} & \leq\left\|\mathcal{N}_{0} G_{1+i t}\right\|_{L^{2}} \leq C\left\|G_{1+i t}\right\|_{L^{2}} \leq C M_{1}\|f\|_{L^{p(\theta)}} .
\end{aligned}
$$

Again standard interpolation gives

$$
\left\|N_{0}^{\eta} G_{\theta}\right\|_{L^{p(\theta)^{\prime}}} \leq C M_{1}^{\theta} M_{0}^{1-\theta}\|f\|_{L^{p(\theta)}},
$$

with $C$ independent of the choice of $\eta$, hence

$$
\left\|\mathcal{N}_{0} G_{\theta}\right\|_{L^{p(\theta)^{\prime}}} \leq C M_{1}^{\theta} M_{0}^{1-\theta}\|f\|_{L^{p(\theta)}} .
$$

Combining (11.15) and (11.18) yields

$$
\left\|\mathcal{N} G_{\theta}\right\|_{L^{p(\theta)^{\prime}}} \leq C M_{1}^{\theta} M_{0}^{1-\theta}\|f\|_{L^{p(\theta)}} .
$$


Then Proposition 8.1 gives an estimate on $\left\|G_{\theta}\right\|_{L^{p(\theta)}}$, yielding

$$
\left\|\psi_{\varepsilon} e^{\varepsilon \Delta}(\lambda I-\Delta)^{s(1-\theta) / 2} R f\right\|_{L^{p(\theta)^{\prime}}} \leq C M_{1}^{\theta} M_{0}^{1-\theta}\|f\|_{L^{p(\theta)}},
$$

with $C$ independent of $\varepsilon \in(0,1]$. Taking $\varepsilon \searrow 0$ gives

$$
(\lambda I-\Delta)^{s(1-\theta) / 2} R f \in L^{p(\theta)^{\prime}}(M),
$$

with norm estimates, implying (11.3) and (11.4).

We illustrate Proposition 11.1 by considering estimates on

$$
S(t)=\frac{\sin t \sqrt{-\Delta}}{\sqrt{-\Delta}} .
$$

In general, if there are no pairs of conjugate points in $M$ (and under some additional technical hypotheses),

$$
\begin{aligned}
& S(t): L^{2}(M) \longrightarrow H^{1,2}(M), \\
& S(t): L^{1}(M) \longrightarrow \mathfrak{h}^{-(n-1) / 2, \infty}(M),
\end{aligned}
$$

where $N=\operatorname{dim} M$. The first mapping property holds by spectral theory and the second by the parametrix construction for solutions to the wave equation; it is this second part that requires the absence of conjugate points. Let us assume we have estimates of the form

$$
\begin{aligned}
\|S(t) f\|_{H^{1,2}} & \leq A_{1}(t)\|f\|_{L^{2}}, \\
\|S(t) f\|_{\mathfrak{h}^{-(n-1) / 2, \infty}} & \leq A_{0}(t)\|f\|_{L^{1}} .
\end{aligned}
$$

It is well known that

$$
\begin{aligned}
M=\mathbb{R}^{n} \Longrightarrow A_{1}(t) & =a(1+|t|), \\
A_{0}(t) & =b_{n}|t|^{-(n-1) / 2} .
\end{aligned}
$$

This estimate for $A_{1}(t)$ is universally valid, by the spectral theorem. On the other hand, for some manifolds with bounded geometry, one can do better. Namely, the following might apply:

$$
\operatorname{Spec}(-\Delta) \subset\left[B^{2}, \infty\right), B>0 \Longrightarrow A_{1}(t)=a_{B} .
$$

While the estimate on $A_{0}(t)$ in (11.25) is typically sharp for $|t| \leq 1$, for general $M$ with bounded geometry (and, say, with sectional curvature $\leq 0)$, sometimes there can be faster decay as $|t| \rightarrow \infty$.

To apply Proposition 11.1, it is convenient to pick $\lambda>K_{0}^{2}$ and consider

$$
R(t)=(\lambda I-\Delta)^{1 / 2} S(t)
$$


Then (11.23)-(11.24) yield

$$
\begin{aligned}
R(t) & : L^{2}(M) \longrightarrow L^{2}(M), \\
R(t) & : L^{1}(M) \longrightarrow \mathfrak{h}^{-(n+1) / 2, \infty}(M),
\end{aligned}
$$

and

$$
\begin{aligned}
\|R(t) f\|_{L^{2}} & \leq C A_{1}(t)\|f\|_{L^{2}}, \\
\|R(t) f\|_{\mathfrak{h}^{-(n+1) / 2, \infty}} & \leq C A_{0}(t)\|f\|_{L^{1}} .
\end{aligned}
$$

Then we can apply Proposition 11.1 to get, for $\theta \in(0,1)$,

$$
R(t): L^{p}(M) \longrightarrow H^{-(1-\theta)(n+1) / 2, p^{\prime}}(M), \quad p=\frac{2}{2-\theta}, p^{\prime}=\frac{2}{\theta},
$$

with operator norm bounded by $C_{\theta} A_{1}(t)^{\theta} A_{0}(t)^{1-\theta}$. Returning to $S(t)$, we have

$$
S(t): L^{p}(M) \longrightarrow H^{1-(1-\theta)(n+1) / 2, p^{\prime}}(M), \quad p=\frac{2}{2-\theta}, p^{\prime}=\frac{2}{\theta},
$$

with

$$
\|S(t) f\|_{H^{1-(1-\theta)(n+1) / 2, p^{\prime}}} \leq C_{\theta} A_{1}(t)^{\theta} A_{0}(t)^{1-\theta}\|f\|_{L^{p}}
$$

For example, we can pick $\theta$ so that

$$
1-(1-\theta) \frac{n+1}{2}=0, \quad \text { i.e., } \theta=\frac{n-1}{n+1},
$$

and obtain

$$
\frac{\sin t \sqrt{-\Delta}}{\sqrt{-\Delta}}: L^{p}(M) \longrightarrow L^{p^{\prime}}(M), \quad p=2 \frac{n+1}{n+3}, p^{\prime}=2 \frac{n+1}{n-1},
$$

with

$$
\left\|\frac{\sin t \sqrt{-\Delta}}{\sqrt{-\Delta}} f\right\|_{L^{p^{\prime}}} \leq C A_{1}(t)^{(n-1) /(n+1)} A_{0}(t)^{2 /(n+1)}\|f\|_{L^{p}} .
$$

In case $M=\mathbb{R}^{n}$, we obtain (with $p, p^{\prime}$ as in (11.34)) the well known result

$$
\left\|\frac{\sin t \sqrt{-\Delta}}{\sqrt{-\Delta}} f\right\|_{L^{p^{\prime}}} \leq C t^{-(n-1) /(n+1)}\|f\|_{L^{p}}
$$

at least for $|t| \leq 1$, but then a simple scaling argument gives (11.36) for all $t$. For non-euclidean manifolds $M$, scaling is not available. In many cases one gets an 
estimate of the form (11.36) valid for $|t| \leq 1$, and in some cases one might get a better estimate for $|t| \geq 1$.

In case $M$ is hyperbolic space $\mathcal{H}^{n}$, results stronger than those obtained in (11.35) via (11.23) are possible, along the following lines. In such a case, (11.26) holds with $B=(n-1) / 2$. One can set

$$
L=\Delta-\left(\frac{n-1}{2}\right)^{2}
$$

write

$$
\frac{\sin t \sqrt{-\Delta}}{\sqrt{-\Delta}}=S_{0}(t)+S_{1}(t), \quad S_{0}(t)=\frac{\sin t \sqrt{-L}}{\sqrt{-L}},
$$

and estimate $\left\|S_{0}(t) f\right\|_{H^{1-s, p^{\prime}}}, \quad s=(1-\theta)(n+1) / 2$, via arguments leading to (11.32) (obtaining stronger estimates), while applying other techniques to estimate $\left\|S_{1}(t) f\right\|_{H^{1-s, p^{\prime}}}$. Work on this will be taken up elsewhere.

\section{A. The space $\operatorname{vmo}(M)$}

Given a Riemannian manifold $M$ with bounded geometry, we define vmo $(M)$ to be the closure in $\operatorname{bmo}(M)$ of the space $C_{*}(M)$ of continuous functions vanishing at infinity. This is parallel to the characterization of $\operatorname{VMO}\left(\mathbb{R}^{n}\right)$, introduced in [Sar], as the closure in $\operatorname{BMO}\left(\mathbb{R}^{n}\right)$ of $C_{*}\left(\mathbb{R}^{n}\right)$. There are equivalent characterizations of $\operatorname{vmo}(M)$, e.g., the closure in $\operatorname{bmo}(M)$ of $C_{0}^{\infty}(M)$. We set

$$
\begin{aligned}
& \|f\|_{\mathrm{vmo}}=\|f\|_{\mathrm{bmo}} \quad \text { if } f \in \operatorname{vmo}(M) \text {, } \\
& \infty \quad \text { if } f \notin \operatorname{vmo}(M) \text {. }
\end{aligned}
$$

It readily follows from Proposition 3,1 that

$$
f \in \operatorname{vmo}(M) \Longrightarrow a f \in \operatorname{vmo}(M)
$$

as long as $a \in L^{\infty}(M) \cap \operatorname{Lip}(M)$, or more generally $a \in L^{\infty}(M) \cap C^{\sigma}(M)$, with $\sigma$ given by (3.23). From here, an argument parallel to the proof of Proposition 3.2 gives:

Proposition A.1. Let $\left\{\varphi_{k}: k \in \mathbb{Z}^{+}\right\}$be a tame partition of unity. Given $f \in$ $L_{\text {loc }}^{1}(M)$, we have

$$
f \in \operatorname{vmo}(M) \Longleftrightarrow \sup _{k}\left\|\varphi_{k} f\right\|_{\mathrm{vmo}}<\infty, \text { and } \lim _{k \rightarrow \infty}\left\|\varphi_{k} f\right\|_{\mathrm{vmo}}=0 .
$$

In [Sar] it was proven that

$$
\operatorname{VMO}\left(\mathbb{R}^{n}\right)^{\prime}=H^{1}\left(\mathbb{R}^{n}\right) .
$$

Our next goal is to prove the following analogue: 
Proposition A.2. If $M$ is a Riemannian manifold with bounded geometry,

$$
\operatorname{vmo}(M)^{\prime}=\mathfrak{h}^{1}(M)
$$

The proof of Proposition 4.1 yields a natural map $\mathcal{I}: \mathfrak{h}^{1}(M) \rightarrow \operatorname{vmo}(M)^{\prime}$, which is clearly one-to-one. To show that $\mathcal{I}$ is surjective, we will construct the inverse $\mathcal{J}: \operatorname{vmo}(M)^{\prime} \rightarrow \mathfrak{h}^{1}(M)$.

Before tackling this, we note that Proposition 4.1 implies there exists $C_{0}=$ $C_{0}(M)$ such that

$$
C_{0}^{-1}\|f\|_{\mathfrak{h}^{1}} \leq \sup \left\{\langle f, g\rangle: g \in \operatorname{bmo}(M),\|g\|_{\text {bmo }} \leq 1\right\} \leq C_{0}\|f\|_{\mathfrak{h}^{1}} .
$$

Part of the content of $(\mathrm{A} .5)$ is that

$$
C_{0}^{-1}\|f\|_{\mathfrak{h}^{1}} \leq \sup \left\{\langle f, g\rangle: g \in \operatorname{vmo}(M),\|g\|_{\mathrm{vmo}} \leq 1\right\} \leq C_{0}\|f\|_{\mathfrak{h}^{1}}
$$

Of course the second inequality in (A.7) follows from its counterpart in (A.6). Our task is to prove the first inequality in (A.7). We start with the case

$$
\mathbb{T}^{n}=\mathbb{R}^{n} / 2 \pi \mathbb{Z}^{n}
$$

Lemma A.3. The result (A.7) holds when $M=\mathbb{T}^{n}$.

Proof. As mentioned, we need only establish the first inequality in (A.7). Fix $f \in \mathfrak{h}^{1}(M)$. Given $\delta>0$, pick $g_{\delta} \in \operatorname{bmo}\left(\mathbb{T}^{n}\right)$ such that

$$
\left\|g_{\delta}\right\|_{\text {bmo }} \leq 1, \quad\|f\|_{\mathfrak{h}^{1}} \leq C_{0}(1+\delta)\left\langle f, g_{\delta}\right\rangle
$$

Now

$$
\begin{aligned}
\left\langle f, e^{\varepsilon \Delta} g_{\delta}\right\rangle & =\left\langle e^{\varepsilon \Delta} f, g_{\delta}\right\rangle \\
& =\left\langle f, g_{\delta}\right\rangle-\left\langle f-e^{\varepsilon \Delta} f, g_{\delta}\right\rangle
\end{aligned}
$$

and, since $C^{\infty}\left(\mathbb{T}^{n}\right)$ is dense in $\mathfrak{h}^{1}\left(\mathbb{T}^{n}\right)$,

$$
\left|\left\langle f-e^{\varepsilon \Delta} f, g_{\delta}\right\rangle\right| \leq C\left\|f-e^{\varepsilon \Delta} f\right\|_{\mathfrak{h}^{1}}\left\|g_{\delta}\right\|_{\mathrm{bmo}} \leq \eta(f, \varepsilon)
$$

where $\eta(f, \varepsilon) \rightarrow 0$ as $\varepsilon \searrow 0$. Hence

$$
\left\langle f, e^{\varepsilon \Delta} g_{\delta}\right\rangle \geq \frac{1}{C_{0}(1+\delta)}\|f\|_{\mathfrak{h}^{1}}-\eta(f, \varepsilon)
$$

Now $e^{\varepsilon \Delta} g_{\delta} \in C^{\infty}\left(\mathbb{T}^{n}\right) \subset \operatorname{vmo}\left(\mathbb{T}^{n}\right)$ for each $\varepsilon>0$, and $\left\|e^{\varepsilon \Delta} g_{\delta}\right\|_{\text {vmo }} \leq 1$. Taking positive $\varepsilon$ and $\delta$ arbitrarily small yields (A.7), for $M=\mathbb{T}^{n}$. 
We now prove Proposition A.2 in case $M=\mathbb{T}^{n}$. Thus, let

$$
\omega: \operatorname{vmo}\left(\mathbb{T}^{n}\right) \longrightarrow \mathbb{R}
$$

be a continuous linear functional. Also set, for $\varepsilon>0$,

$$
\omega_{\varepsilon}(f)=\omega\left(e^{\varepsilon \Delta} f\right) .
$$

Clearly we have a unique $g_{\varepsilon} \in C^{\infty}\left(\mathbb{T}^{n}\right)$ such that

$$
\omega_{\varepsilon}(f)=\left\langle f, g_{\varepsilon}\right\rangle=\int_{\mathbb{T}^{n}} f g_{\varepsilon} d x
$$

By Lemma A.3, we have

$$
\left\|g_{\varepsilon}\right\|_{\mathfrak{h}^{1}} \leq C_{0}\left\|\omega_{\varepsilon}\right\| \leq C_{0}\|\omega\|<\infty
$$

Now we have (perhaps passing to a subsequence $\varepsilon=\varepsilon_{j} \searrow 0$ )

$$
g_{\varepsilon} \longrightarrow g \text { weak }^{*} \text { in } \mathcal{M}\left(\mathbb{T}^{n}\right)
$$

where $\mathcal{M}\left(\mathbb{T}^{n}\right)$ denotes the space of finite Borel measures on $\mathbb{T}^{n}$, and

$$
\omega(f)=\langle f, g\rangle, \quad \forall f \in C\left(\mathbb{T}^{n}\right) .
$$

To conclude the proof of Proposition A.2 for $M=\mathbb{T}^{n}$, it remains to show that

$$
g \in \mathfrak{h}^{1}\left(\mathbb{T}^{n}\right)
$$

To see this, note that

$$
\mathcal{G}^{\rho} g(x) \nearrow \mathcal{G}^{b} g(x) \text { as } \rho \searrow 0
$$

where, for $\rho \in(0,1)$,

$$
\begin{aligned}
\mathcal{G}^{\rho} g(x) & =\sup _{r \in[\rho, 1]} \mathcal{G}^{r} g(x) \\
& =\sup \left\{|\langle\varphi, g\rangle|: \varphi \in \bigcup_{r \in[\rho, 1]} \mathcal{F}\left(B_{r}(x)\right)\right\},
\end{aligned}
$$

with $\mathcal{F}\left(B_{r}(x)\right)$ as in (2.3). Since $\cup_{x \in \mathbb{T}^{n}} \cup_{r \in[\rho, 1]} \mathcal{F}\left(B_{r}(x)\right)$ is a relatively compact subset of $C\left(\mathbb{T}^{n}\right)$ for each $\rho \in(0,1)$, we have

$$
\mathcal{G}^{\rho} g_{\varepsilon}(x) \rightarrow \mathcal{G}^{\rho} g(x) \text { as } \varepsilon \rightarrow 0
$$


uniformly in $x$, for each $\rho>0$. Hence, by (A.16),

$$
\left\|\mathcal{G}^{\rho} g\right\|_{L^{1}} \leq C_{0}\|\omega\|
$$

We then deduce from (A.20) that

$$
\mathcal{G}^{b} g \in L^{1}\left(\mathbb{T}^{n}\right)
$$

This implies the desired result (A.19).

We proceed with the proof of Proposition A.2 for general $M$ with bounded geometry. To set things up, bring in a tame partition of unity $\left\{\varphi_{k}: k \in \mathbb{Z}^{+}\right\}$, $\varphi_{k} \in C_{0}^{\infty}\left(B_{1}\left(p_{k}\right)\right)$, as defined in (1.25)-(1.27). As in the proof of Proposition 2.2, partition $\mathbb{Z}^{+}$into $K_{1}=K_{1}(M)$ sets $\mathcal{S}_{1}, \ldots, \mathcal{S}_{K_{1}}$ such that $j, k \in \mathcal{S}_{\nu}, j \neq k \Rightarrow$ $d\left(p_{j}, p_{k}\right) \geq 20$, and for each $\nu \in\left\{1, \ldots, K_{1}\right\}$, set

$$
T_{\nu} f=\sum_{k \in \mathcal{S}_{\nu}} \varphi_{k} f
$$

We have

$$
T_{\nu}: \operatorname{vmo}(M) \longrightarrow \operatorname{vmo}(M), \quad\left\|T_{\nu} f\right\|_{\mathrm{vmo}} \leq C\|f\|_{\mathrm{vmo}}, 1 \leq \nu \leq K_{1} .
$$

Of course, $f=\sum_{\nu} T_{\nu} f$.

Now let $\omega: \operatorname{vmo}(M) \rightarrow \mathbb{R}$ be a continuous linear functional. We want to define $\mathcal{J} \omega \in \mathfrak{h}^{1}(M)$. To do this it suffices to define $\mathcal{J} \omega_{\nu}$ for each $\nu \in\left\{1, \ldots, K_{1}\right\}$, where $\omega_{\nu}=\omega \circ T_{\nu}$. Also define $\omega_{k}: \operatorname{vmo}(M) \rightarrow \mathbb{R}$ by

$$
\omega_{k}(f)=\omega\left(\varphi_{k} f\right) \text {. }
$$

Using the identification of $B_{2}\left(p_{k}\right)$ with $B_{2}(0) \subset \mathbb{R}^{n}$ via $\operatorname{Exp}_{p_{k}}$, and then identifying $B_{2}(0) \subset \mathbb{R}^{n}$ with $B_{2}(0) \subset \mathbb{T}^{n}$, we can use the special case just proven to write

$$
\omega_{k}(f)=\left\langle f, g_{k}\right\rangle
$$

where $g_{k} \in \mathfrak{h}^{1}\left(\mathbb{T}^{n}\right)$ has support in $B_{2}(0)$. Multiplying by the volume form, we can identify $g_{k}$ with an element of $\mathfrak{h}^{1}(M)$, supported in $B_{2}\left(p_{k}\right)$ :

$$
g_{k} \in \mathfrak{h}^{1}(M), \quad \operatorname{supp} g_{k} \subset B_{2}\left(p_{k}\right) .
$$

We next claim that

$$
\omega_{\nu}(f)=\left\langle f, g_{\nu}\right\rangle
$$

with

$$
g_{\nu}=\sum_{k \in \mathcal{S}_{\nu}} g_{k} \in \mathfrak{h}^{1}(M)
$$


Note that the terms in this sum have widely disjoint supports, and if $\widetilde{\mathcal{S}}_{\nu} \subset \mathcal{S}_{\nu}$ is any finite subset, then

$$
\tilde{g}_{\nu}=\sum_{\widetilde{\mathcal{S}}_{\nu}} g_{k} \Rightarrow \sum_{k \in \widetilde{\mathcal{S}}_{\nu}} \omega\left(\varphi_{k} f\right)=\left\langle f, \tilde{g}_{\nu}\right\rangle, \quad\left\|\tilde{g}_{\nu}\right\|_{\mathfrak{h}^{1}}=\sum_{k \in \widetilde{\mathcal{S}}_{\nu}}\left\|g_{k}\right\|_{\mathfrak{h}^{1}}
$$

Using Lemma A.3, we can produce $f_{k} \in \operatorname{vmo}(M)$ such that

$$
\operatorname{supp} f_{k} \subset B_{2}\left(p_{k}\right), \quad\left\|f_{k}\right\|_{\mathrm{vmo}} \leq C_{1}, \quad\left\langle f_{k}, g_{k}\right\rangle \geq\left\|g_{k}\right\|_{\mathfrak{h}^{1}}
$$

with $C_{1}=C_{1}(M)<\infty$. Then, given any finite subset $\widetilde{\mathcal{S}}_{\nu} \subset \mathcal{S}_{\nu}$,

$$
\tilde{f}_{\nu}=\sum_{k \in \widetilde{\mathcal{S}}_{\nu}} f_{k} \Longrightarrow\left\|\tilde{f}_{\nu}\right\|_{\mathrm{vmo}} \leq C_{1}
$$

and

$$
\begin{aligned}
\sum_{k \in \widetilde{\mathcal{S}}_{\nu}}\left\|g_{k}\right\|_{\mathfrak{h}^{1}} & \leq \sum_{k \in \widetilde{\mathcal{S}}_{\nu}}\left\langle f_{k}, g_{k}\right\rangle \\
& =\left\langle\tilde{f}_{\nu}, \tilde{g}_{\nu}\right\rangle \\
& =\omega_{\nu}\left(\tilde{f}_{\nu}\right) \leq C_{2}<\infty
\end{aligned}
$$

the last inequality by (A.34). This implies

$$
g_{\nu}=\sum_{k \in \mathcal{S}_{\nu}} g_{k} \in \mathfrak{h}^{1}(M)
$$

and we have $g_{\nu}=\mathcal{J} \omega_{\nu}$, finishing the proof of Proposition A.2.

We next examine the action of pseudodifferential operators on $\operatorname{vmo}(M)$. The following result complements Proposition 6.7.

Proposition A.4. Given $K_{0}$ as in (6.16),

$$
W>K_{0}, P \in \Psi_{W}^{0}(M) \Longrightarrow P: \operatorname{vmo}(M) \rightarrow \operatorname{vmo}(M) .
$$

Proof. As in $\S 6$, write $P=P^{\#}+P^{b}$, with $P^{\#} \in \Psi_{\#}^{0}(M), P^{b} \in \Psi_{W}^{-\infty}(M)$. One readily verifies the following:

$$
P^{\#}: C_{0}^{\infty}(M) \longrightarrow C_{0}^{\infty}(M), \quad P^{b}: C_{0}^{\infty}(M) \longrightarrow C_{*}(M)
$$

SO

$$
P: C_{0}^{\infty}(M) \longrightarrow C_{*}(M)
$$


The result (A.37) follows from this together with the boundedness on $\operatorname{bmo}(M)$ given in (6.37).

\section{B. The operator class $\widetilde{\Psi}_{W}^{m}(M)$}

Given $m \in \mathbb{R}, W>0$, we define an operator class $\widetilde{\Psi}_{W}^{m}(M)$, smaller than $\Psi_{W}^{m}(M)$, which was defined in $\S 6$, and discuss some properties. Parallel to (6.27)-(6.31), we set

$$
\widetilde{\Psi}_{W}^{m}(M)=\left\{P^{\#}+P^{b}: P^{\#} \in \Psi_{\#}^{m}(M), P^{b} \in \widetilde{\Psi}_{W}^{-\infty}(M)\right\}
$$

where $\Psi_{\#}^{m}(M)$ is as in $(6.23)-(6.25)$, and we say $P^{b} \in \widetilde{\Psi}_{W}^{-\infty}(M)$ provided it has the form

$$
P^{b} f(x)=\int_{M} k^{b}(x, y) f(y) d V(y),
$$

where $k^{b} \in C^{\infty}(M \times M)$ satisfies, for each $x, y \in M, r \in(0, \infty), \ell \in \mathbb{Z}^{+}$,

$$
\begin{aligned}
\left\|k^{b}(\cdot, y)\right\|_{L^{2}\left(M \backslash B_{r}(y)\right)} & \leq C_{\ell}\langle r\rangle^{-\ell} e^{-W r}, \\
\left\|k^{b}(x, \cdot)\right\|_{L^{2}\left(M \backslash B_{r}(x)\right)} & \leq C_{\ell}\langle r\rangle^{-\ell} e^{-W r},
\end{aligned}
$$

with similar estimates on all $x$ and $y$-derivatives of $k^{b}(x, y)$.

It is easy to check that $\widetilde{\Psi}_{W}^{-\infty}(M) \subset \Psi_{W}^{-\infty}(M)$, and hence $\widetilde{\Psi}_{W}^{m}(M) \subset \Psi_{W}^{m}(M)$. The following result improves Proposition 6.6.

Proposition B.1. For $W>0, m \in \mathbb{R}$,

$$
\Phi \in \mathcal{S}_{W}^{m} \Longrightarrow \Phi(\sqrt{-\Delta}) \in \widetilde{\Psi}_{W}^{m}(M) .
$$

More generally, if

$$
\operatorname{Spec}(-\Delta) \subset\left[B^{2}, \infty\right)
$$

and $L=\Delta+B^{2}$, then

$$
\Phi \in \mathcal{S}_{W}^{m} \Longrightarrow \Phi(\sqrt{-L}) \in \widetilde{\Psi}_{W}^{m}(M)
$$

The proof of this is given in (1.8)-(1.13) of [T3].

In light of this, the following result has stronger consequences for $L^{p}$ estimates on $\Phi(\sqrt{-\Delta})$ than Proposition 6.7 does. Recall the volume estimate $(6.16)$ :

$$
\operatorname{Vol}\left(B_{r}(p)\right) \leq C_{0}(1+r)^{\mu_{0}} e^{K_{0} r}, \quad \forall p \in M, r \in(0, \infty) .
$$


Proposition B.2. If $W \geq K_{0} / 2$, then

$$
P^{b} \in \widetilde{\Psi}_{W}^{-\infty}(M) \Longrightarrow P: L^{p}(M) \rightarrow L^{p}(M), \quad \forall p \in[1, \infty] .
$$

Hence

$$
\begin{aligned}
P \in \widetilde{\Psi}_{W}^{0}(M) \Longrightarrow & P: L^{p}(M) \rightarrow L^{p}(M), \quad \forall p \in(1, \infty), \\
P: \mathfrak{h}^{1}(M) & \rightarrow L^{1}(M), \\
P & : L^{\infty}(M) \rightarrow \operatorname{bmo}(M) .
\end{aligned}
$$

Proof. It suffices to prove (B.8), since Proposition 6.5 then gives (B.9). If $k^{b}(x, y)$ is the integral kernel of $P^{b}$, to prove (B.8) it suffices to show that

$$
\begin{aligned}
& \sup _{y} \int_{M}\left|k^{b}(x, y)\right| d V(x)<\infty, \quad \text { and } \\
& \sup _{x} \int_{M}\left|k^{b}(x, y)\right| d V(y)<\infty .
\end{aligned}
$$

We estimate the first integral in (B.10) by dividing $M$ into shells

$$
A_{j}(y)=\{x \in M: j \leq d(x, y) \leq j+1\} .
$$

We have the following estimate:

$$
\begin{aligned}
\int_{M}\left|k^{b}(x, y)\right| d V(x) & =\sum_{j \geq 0} \int_{A_{j}(y)}\left|k^{b}(x, y)\right| d V(x) \\
& \leq \sum_{j \geq 0}\left(\operatorname{Vol} A_{j}(y)\right)^{1 / 2}\left\|k^{b}(\cdot, y)\right\|_{L^{2}\left(A_{j}(y)\right)} \\
& \leq C \sum_{j \geq 0}\langle j\rangle^{\mu_{0} / 2} e^{j K_{0} / 2}\left\|k^{b}(\cdot, y)\right\|_{L^{2}\left(A_{j}(y)\right)} .
\end{aligned}
$$

Bringing in (B.3), we have

$$
\left\|k^{b}(\cdot, y)\right\|_{L^{2}\left(A_{j}(y)\right)} \leq C_{\ell}\langle j\rangle^{-\ell} e^{-j W},
$$

and taking $\ell>\mu_{0} / 2+1$ yields the first bound in (B.10), as long as $W \geq K_{0} / 2$. The second bound in (B.10) is proven similarly.

Propositions B.1-B.2 yield the following improvement over Proposition 6.7. This result can be compared with Theorem 10.2 of [CMM]. 
Corollary B.3. If the volume estimate (B.7) holds, and if (B.5) holds and $L=$ $\Delta+B^{2}$, then

$$
\begin{aligned}
\Phi \in \mathcal{S}_{W}^{0}, W \geq \frac{K_{0}}{2} \Longrightarrow & \Phi(\sqrt{-L}): L^{p}(M) \rightarrow L^{p}(M), \quad p \in(1, \infty) \\
& \Phi(\sqrt{-L}): \mathfrak{h}^{1}(M) \rightarrow L^{1}(M) \\
& \Phi(\sqrt{-L}): L^{\infty}(M) \rightarrow \operatorname{bmo}(M)
\end{aligned}
$$

Regarding $L^{p}$-estimates, (B.14) plus an application of the Stein interpolation theorem yields the following (Theorem 1.6 of [T3]):

Proposition B.4. If $\Phi \in \mathcal{S}_{W}^{0}$, then

$$
\Phi(\sqrt{-L}): L^{p}(M) \longrightarrow L^{p}(M)
$$

provided

$$
p \in(1, \infty), \quad \text { and } W \geq\left|\frac{1}{p}-\frac{1}{2}\right| \cdot K_{0}
$$

We finish with the following improvement of Proposition B.2 and Corollary B.3.

Proposition B.5. If $W \geq K_{0} / 2$, then

$$
\begin{aligned}
P^{b}: \widetilde{\Psi}_{W}^{-\infty}(M) \Longrightarrow P^{b}: \operatorname{bmo}(M) & \rightarrow L^{\infty}(M), \\
P^{b}: L^{1}(M) & \rightarrow \mathfrak{h}^{1}(M) .
\end{aligned}
$$

Hence

$$
\begin{aligned}
& P: \widetilde{\Psi}_{W}^{0}(M) \Longrightarrow P: \operatorname{bmo}(M) \rightarrow \operatorname{bmo}(M), \\
& P: \mathfrak{h}^{1}(M) \rightarrow \mathfrak{h}^{1}(M)
\end{aligned}
$$

Consequently, in the setting of Corollary B.3,

$$
\begin{aligned}
\Phi \in \mathcal{S}_{W}^{0} \Longrightarrow \Phi(\sqrt{-L}): \operatorname{bmo}(M) \rightarrow \operatorname{bmo}(M), \\
\Phi(\sqrt{-L}): \mathfrak{h}^{1}(M) \rightarrow \mathfrak{h}^{1}(M) .
\end{aligned}
$$

Proof. We prove the first part of (B.16). This readily yields

$$
P^{b}: \operatorname{vmo}(M) \longrightarrow C_{*}(M)
$$

the latter space consisting of continuous functions on $M$ vanishing at infinity, and the second part follows by duality. From here, (B.17) and (B.18) follow by the same arguments as used above. 
To proceed, take $f \in \operatorname{bmo}(M)$. Pick $\lambda>K_{0}^{2}$, and write

$$
P^{b} f=P^{b}(\lambda I-\Delta)\left((\lambda I-\Delta)^{-1} f\right) .
$$

By Proposition 10.1 and the characterization (10.7) of $\mathfrak{h}^{2, \infty}(M)$, we have

$$
f \in \operatorname{bmo}(M) \Longrightarrow(\lambda I-\Delta)^{-1} f \in L^{\infty}(M) .
$$

On the other hand, since the integral kernel of $P^{b}(\lambda I-\Delta)$ is $\left(\lambda I-\Delta_{y}\right) k^{b}(x, y)$, it is clear from the definition that

$$
P^{b} \in \widetilde{\Psi}_{W}^{-\infty}(M) \Longrightarrow P^{b}(\lambda I-\Delta) \in \widetilde{\Psi}_{W}^{-\infty}(M) .
$$

Thus Proposition B.2 gives

$$
P^{b}(\lambda I-\Delta): L^{\infty}(M) \longrightarrow L^{\infty}(M),
$$

and the proof is done.

\section{Further results for symmetric spaces of noncompact type}

A symmetric space of noncompact type is a Riemannian manifold $M=G / K$, where $G$ is a semisimple Lie group of noncompact type and $K$ a maximal compact subgroup. Examples include hyperbolic space $\mathcal{H}^{n}$, with constant sectional curvature -1 , amongst others. (However, this definition excludes Euclidean space.) We refer to [Hel] for basic material; basic results are also summarized in $\S 2$ of [T3]. Without going into details, we mention the following key fact: there exists a positive quantity, denoted $|\rho|^{2}$, with the property that

$$
\operatorname{Spec}(-\Delta)=\left[|\rho|^{2}, \infty\right) \quad \text { on } L^{2}(M)
$$

and

$$
\operatorname{Vol} B_{r}(p) \sim C r^{\beta} e^{2|\rho| r}, \quad r \rightarrow \infty,
$$

for some $\beta \in(0, \infty)$. Cf. [T3], (2.2) and (2.9). When $M=\mathcal{H}^{n},|\rho|=(n-1) / 2$. Now, if we set

$$
L=\Delta+|\rho|^{2},
$$

so Spec $(-L)=[0, \infty)$ on $L^{2}(M)$, we can apply Proposition B.4 to deduce that, for $p \in(1, \infty)$,

$$
\Phi \in \mathcal{S}_{W}^{0}, W>\left|\frac{2}{p}-1\right| \cdot|\rho| \Longrightarrow \Phi(\sqrt{-L}): L^{p}(M) \rightarrow L^{p}(M) .
$$

Using this, we can establish the following variant of the fact that

$$
(\lambda I-\Delta)^{m / 2}: H^{s, p}(M) \longrightarrow H^{s-m, p}(M),
$$

for $s, m \in \mathbb{R}, p \in(1, \infty)$, given $\lambda>0$ sufficiently large, which was proven in $\S 10$, in the setting of general manifolds with bounded geometry. 
Proposition C.1. If $M$ is a symmetric space of noncompact type, then for $s, m \in$ $\mathbb{R}, p \in(1, \infty)$,

$$
(-\Delta)^{m / 2}: H^{s, p}(M) \longrightarrow H^{s-m, p}(M)
$$

REMARK. This fails when $M=\mathbb{R}^{n}$.

Proof. In light of the results of $\S 10,($ C.6) is equivalent to the assertion that, for $\lambda>0$ sufficiently large,

$$
(\lambda I-\Delta)^{(s-m) / 2}(-\Delta)^{m / 2}(\lambda I-\Delta)^{-s / 2}: L^{p}(M) \longrightarrow L^{p}(M) .
$$

We can write this operator as

$$
\left(\lambda I+|\rho|^{2}-L\right)^{(s-m) / 2}\left(|\rho|^{2}-L\right)^{m / 2}\left(\lambda I+|\rho|^{2}-L\right)^{-s / 2}=\Phi(\sqrt{-L}),
$$

where $\Phi(\zeta)=\left(\lambda+|\rho|^{2}+\zeta^{2}\right)^{-m / 2}\left(|\rho|^{2}+\zeta^{2}\right)^{m / 2}$, and we see that

$$
\Phi \in \mathcal{S}_{W}^{0}, \quad \forall W<|\rho|
$$

Now for each $p \in(1, \infty),|2 / p-1|<1$, so (C.7) follows from (C.4).

Proposition C.1 interfaces with results of $\S 11$ as follows. As stated there, for

$$
\theta \in(0,1), \quad p=\frac{2}{2-\theta}, \quad p^{\prime}=\frac{2}{\theta}, \quad s=(1-\theta) \frac{n+1}{2}
$$

we obtain in various circumstances estimates of the form

$$
\left\|\frac{\sin t \sqrt{-\Delta}}{\sqrt{-\Delta}}\right\|_{H^{1-s, p^{\prime}}} \leq \psi_{\theta}(t)\|f\|_{L^{p}}
$$

known as dispersive estimates. Similar estimates yield

$$
\|\cos t \sqrt{-\Delta} f\|_{H^{-s, p^{\prime}}} \leq A_{1} \psi_{\theta}(t)\|f\|_{L^{p}}
$$

Obtaining such estimates, e.g., for $M=\mathcal{H}^{n}$ involves, amongst other things, rather explicit formulas for (distributional) integral kernels of these operators. Such explicit formulas are lacking for the opeators $\sin t \sqrt{-\Delta}$; however applying Proposition C.1 to (C.11) gives

$$
\|\sin t \sqrt{-\Delta} f\|_{H^{-s, p^{\prime}}} \leq A_{2} \psi_{\theta}(t)\|f\|_{L^{p}}
$$


and putting (C.12)-(C.13) together gives for $e^{i t \sqrt{-\Delta}}=\cos t \sqrt{-\Delta}+i \sin t \sqrt{-\Delta}$ the estimate

$$
\left\|e^{i t \sqrt{-\Delta}} f\right\|_{H^{-s, p^{\prime}}} \leq\left(A_{1}+A_{2}\right) \psi_{\theta}(t)\|f\|_{L^{p}}
$$

in case $M$ is a symmetric space of noncompact type. Such an estimate is a convenient variant of (C.11) for the purpose of passing from dispersive estimates to Strichartz estimates. This matter will be pursued elsewhere.

\section{References}

[CMM] A. Carbonaro, G. Mauceri, and S. Meda, $H^{1}$ and BMO for certain nondoubling measured metric spaces, Preprint, 2008.

[CKS] D. Chang, S. Krantz, and E. Stein, $H^{p}$ theory on a smooth domain in $\mathbb{R}^{N}$ and applications to partial differential equations, J. Funct. Anal. 114 (1993), 286-347.

[CGT] J. Cheeger, M. Gromov, and M. Taylor, Finite propagation speed, kernel estimates for functions of the Laplace operator, and the geometry of complete Riemannian manifolds, J. Diff. Geom. 17 (1982), 15-53.

[CW1] R. Coifman and G. Weiss, Analyse harmonique non-commutative sur certains espaces homogenes, LNM \#242, Springer-Verlag, New York, 1971.

[CW2] R. Coifman and G. Weiss, Extensions of Hardy spaces and their use in analysis, Bull. AMS 83 (1977), 569-645.

[FS] C. Fefferman and E. Stein, $H^{p}$ spaces of several variables, Acta Math. 129 (1972), 137-193.

[G] D. Goldberg, A local version of real Hardy spaces, Duke Math. J. 46 (1979), $27-42$.

[Hel] S. Helgason, Lie Groups and Geometric Analysis, Academic Press, New York, 1984.

[I] A. Ionescu, Fourier integral operators on noncompact symmetric spaces of real rank one, J. Funct. Anal. 174 (2000), 274-300.

[JN] F. John and L. Nirenberg, On functions of bounded mean oscillation, Comm. Pure Appl. Math. 24 (1961), 415-426.

[MT] M. Mitrea and M. Taylor, Potential theory in Lipschitz domains in Riemannian manifolds: $L^{p}$, Hardy, and Hölder space results, Comm. in Anal. and Geom. 9 (2001), 369-421.

[Rei] H. Reimann, Functions of bounded mean oscillation and quasiconformal mappings, Comment. Math. Helv. 49 (1974), 260-276.

[Sar] D. Sarason, Functions of vanishing mean oscillation, TAMS 207 (1975), 391-405.

[Sem] S. Semmes, A primer on Hardy spaces, and some remarks on a theorem of Evans and Müller, Comm. PDE 19 (1994), 277-319.

[St0] E. Stein, Topics in Harmonic Analysis Related to the Littlewood-Paley Theory, Princeton Univ. Press, Princeton, NJ, 1970. 
[St] E. Stein, Harmonic Analysis, Princeton Univ. Press, Princeton, NJ, 1993.

[Str1] R. Strichartz, The Hardy space $H^{1}$ on manifolds and submanifolds, Canad. J. Math. 24 (1972), 915-925.

[Str2] R. Strichartz, Analysis of the Laplacian on the complete Riemannian manifold, J. Funct. Anal. 52 (1983), 48-79.

[T1] M. Taylor, Fourier integral operators and harmonic analysis on compact manifolds, Proc. Symp. Pure Math. 35 (Part 2) (1979), 115-136.

[T2] M. Taylor, Pseudodifferential Operators, Princeton Univ. Press, Princeton, NJ, 1981.

[T3] M. Taylor, $L^{p}$ estimates on functions of the Laplace operator, Duke Math. J. 58 (1989), 773-793.

[Tri] H. Triebel, Theory of Function Spaces II, Birkhauser, Boston, 1992. 